\title{
Morphological diagnostic characters of Isodon (Lamiaceae) in Korea
}

\author{
Younju Ma and Sangtae Kim* \\ Department of Biology and Basic Science Institute, Sungshin Women's University, Seoul 142-732, Korea \\ (Received 11 September 2014; Revised 7 October 2014; Accepted 25 November 2014)

\section{한국산 산박하속(꿀풀과)의 외부형태학적 식별 형질} \\ 마윤주 · 김상태* \\ 성신여자대학교 생물학과, 기초과학연구소
}

\begin{abstract}
Although a few recent taxonomic studies focusing on infraspecific taxa in each species of Isodon have been reported, a detailed taxonomic revision of the Korean Isodon taxa has yet to be performed. We investigated the morphological characters of Korean Isodon based on approximately 600 herbarium sheets from major herbaria in Korea and Japan. We identified characters which distinguish seven Korean Isodon taxa and created a key to Korean Isodon based on the results from this study and on recent studies of the Korean Isodon taxa. The following unique characters for several taxa were recognized: 1) dense non-glandular hairs in stems for I. inflexus var. canescens (over 70 $\mathrm{ea} / \mathrm{mm}$ on one side of the stem), 2) dense glandular hairs on the abaxial surface of the leaf for $I$. serra (over $40 \mathrm{ea} / \mathrm{mm}^{2}$ ), 3) protruding pistils and stamens from the corolla with the length of the protruding part longer than that of the lower lobe of corolla for I. japonicus, and 4) the presence of non-glandular hairs on the entire surface of the fruit of $I$. inflexus var. microphyllus. Based on this study, we recognized that there are four species, two varieties, and one forma of Isodon taxa in Korea.
\end{abstract}

Keywords: Isodon, morphological character, key, electron microscopy

적 요: 한국산 산박하속(Isodon)에 대하여 최근 몇몇 종에 있어서는 종내 분류군의 구분에 대한 연구가 이루어 진 바 있지만, 현재까지 한국산 종들의 형태학적 형질에 대한 종합적 고찰은 이루어진 바는 없다. 본 연구에서 는 지금까지 한반도에 자생한다고 보고된 분류군들에 대하여 한국 및 일본의 주요 표본관으로부터 대여한 약 600 여점의 표본을 조사하여 외부형태학적 형질들 도출해 내고, 각각의 형질에 대한 형질상태의 유형을 파악하 여 도해하였다. 그 결과 한국산 산박하속은 4 종 2 변종 1 품종으로 정리되었고, 한국산 분류군들을 구분할 수 있 는 기존의 문헌상의 식별형질들을 재확인 하였으며, 또한 새로운 식별형질들을 제시하였다: 1) 줄기에 있는 털 의 높은 밀도(줄기 한쪽 면에 70개/mm 이상)는 털산박하(I. inflexus var. canescens)를 다른 분류군들로 부터 구분 하는 형질이었고, 2) 잎 뒷면의 선모의 높은 밀도( 40 개 $/ \mathrm{mm}^{2}$ 이상)는 I. serra와 다른 분류군을 구분할 수 있는 형 질이었으며, 3) 화관에 있어서 하순으로부터 암술과 수술이 하순길이 이상으로 돌출하는 것은 I. japonicus 만의 특징이었으며, 4) 과실표면 전체에 비선모가 존재하는 것은 I. inflexus var. microphyllus만의 식별형질이었다.

주요어: 산박하속, 형태형질, 검색표, 전자현미경

Isodon (Schrad. ex Benth.) Spach (산박하속)은 꿀풀과의 최 근 분류체계인 Harley et al. (2004)의 분류체계상 subfamily

\footnotetext{
*Author for correspondence: amborella@sungshin.ac.kr
}

http://www.pltaxa.or.kr

Copyright (C) 2014 the Korean Society of Plant Taxonomists
Nepetoideae에 속하며, 세계적으로 약 100 종 정도가 알려 져 있는데, 열대 또는 아열대의 아시아 지역에 주로 분포 하며 아프리카에도 소수가 분포하고, 산비탈, 숲의 골짜 기, 강기슭이나 길가에 서식하는 다년생 초본이다 (Mabberley, 1987; Li, 1988; Maki et al., 2010; Zhong et al., 2010). Isodon은 꿀풀과 내의 근연 분류군인 Siphocranion 
Kudo, Skapanthus C. Y. Wu \& H. W. Li, Plectranthus L'Hér와 잘 구분된다: Isodon이 동일한(또는 거의 동일한) 크기의 꽃 받침 열편을 갖고 있는 것에 반해 Plectranthus는 한 개의 큰 꽃받침 열편과 동일한 네 개의 작은 꽃받침 열편을 갖고 있 고, 수술이 화관의 입구까지 이합된 Siphocranion에 비해 Isodon은 덜 이합되며, 화사의 기부가 팽대한 Skapanthus에 비해 Isodon은 전체가 동일한 굵기의 화사를 갖고 있다. 그 러나 몇몇 학자들은 광의의 개념으로(sensu lato) 위의 네 속 을 Plectranthus 한 속으로 인식하기도 한다( $(\mathrm{Li}, 1988)$.

한국산 Isodon에 관련된 분류군들은 Ocymum Mill., Amethystanthus Nakai, Plectranthus, Scutellaria L.에 속한 분 류군들로 최초 발표된 이래 학자들마다 이들에 대한 속의 인식과 종 내 분류군들의 인식을 매우 달리하고 있다 (Table 1). 학자들은 이들 분류군들을 Plectranthus (Bentham, 1836; Koidzumi, 1929; Lee, W. T., 1996; Maximowicz, 1875; Nakai, 1911, 1921), Amethystanthus (Nakai 1934), Isodon (Kudo, 1929; Lee, 1985; Korea National Arboretum and the Plant Taxonomic Society of Korea, 2007; Suh et al., 2007; Kim and Ma, 2011, 2013), 또는 Rabdosia (Hara, 1927; Lee, Y. N., 1996)에 속하는 분류군으로 각각 인 식한 바 있다(Table 1). 그러나 최근 Li (1988)는 이들 속들 에 대한 분류학적 재고찰을 통해 Plectranthus의 세 개의 절 인 sect. Isodon Schrad. ex Benth., sect. Pyramidium Benth.,
Table 2. Collection data for a scanning electron microscophic study of trichomes in Korean Isodon.

\begin{tabular}{|c|c|}
\hline Taxa & Locality \\
\hline I. excisus & $\begin{array}{l}\text { Gangwon-do: Mt. Gwangdeok } \\
\text { (Jul. 6, 2009), M. Kong et al. s. } n .(\mathrm{KH})\end{array}$ \\
\hline I. inflexus var. inflexus & $\begin{array}{l}\text { Chungcheongbuk-do: Mt. Worak } \\
\text { (Aug. 23, 2010), Y. Ma } 2010085 \text { (SWU) }\end{array}$ \\
\hline I. inflexus var. canescens & $\begin{array}{l}\text { Jeju-do: Chujado (unknwon), } \\
\text { T. Lee s. } n \text {. (SNUA) }\end{array}$ \\
\hline I. inflexus var. microphyllus & $\begin{array}{l}\text { Jeollanam-do: Bogildo } \\
\text { (Aug. 13, 1964), T. Lee s. n. (SNUA) }\end{array}$ \\
\hline I. japonicus & $\begin{array}{l}\text { Gyeonggi-do: Mt. Jeonggwang } \\
\text { (Oct. 10, 2010), Y. Ma } 2010069 \text { (SWU) }\end{array}$ \\
\hline I. serra & $\begin{array}{l}\text { Jeollanam-do: Gwangju } \\
\text { (Aug. 26, 2001), E.-S. Jeon et al. s. n. }(\mathrm{KH})\end{array}$ \\
\hline
\end{tabular}

sect. Amethystoides Benth.를 Plectranthus로부터 분리하여 하나의 속인 Isodon으로 인식하여 혼란이 있어온 속명들의 이명관계를 정리하였고, 또한 Isodon 내에 네 개의 절들(sect. Pyramidium, sect. Isodon, sect. Melissoides, sect. Amethystoides) 을 인식한 바 있다. 이들 네 개의 절들을 구분하는 특징은 sect. Pyramidium은 꽃받침이 곧은데 반해 sect. Isodon과 sect. Melissoides는 꽃받침이 굽었으며, sect. Pyramidium은 꽃받침 이 통상 또는 통상종형이고 피라미드형 원추화서를 갖는

Table 1. Nomenclatural history of the Korean Isodon.

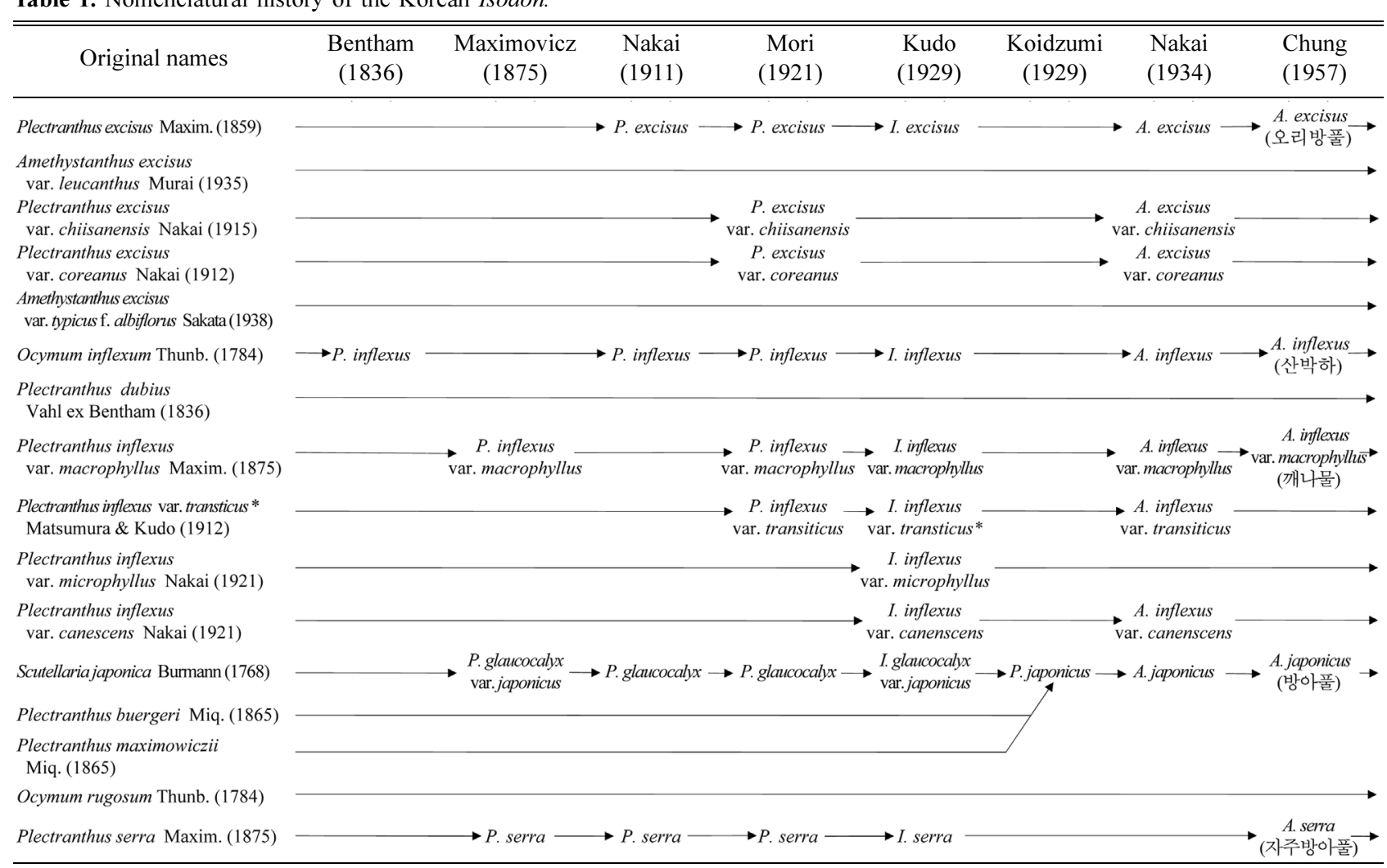

*"transticus" was a type of "transiticus" 
Table 1. Continued.

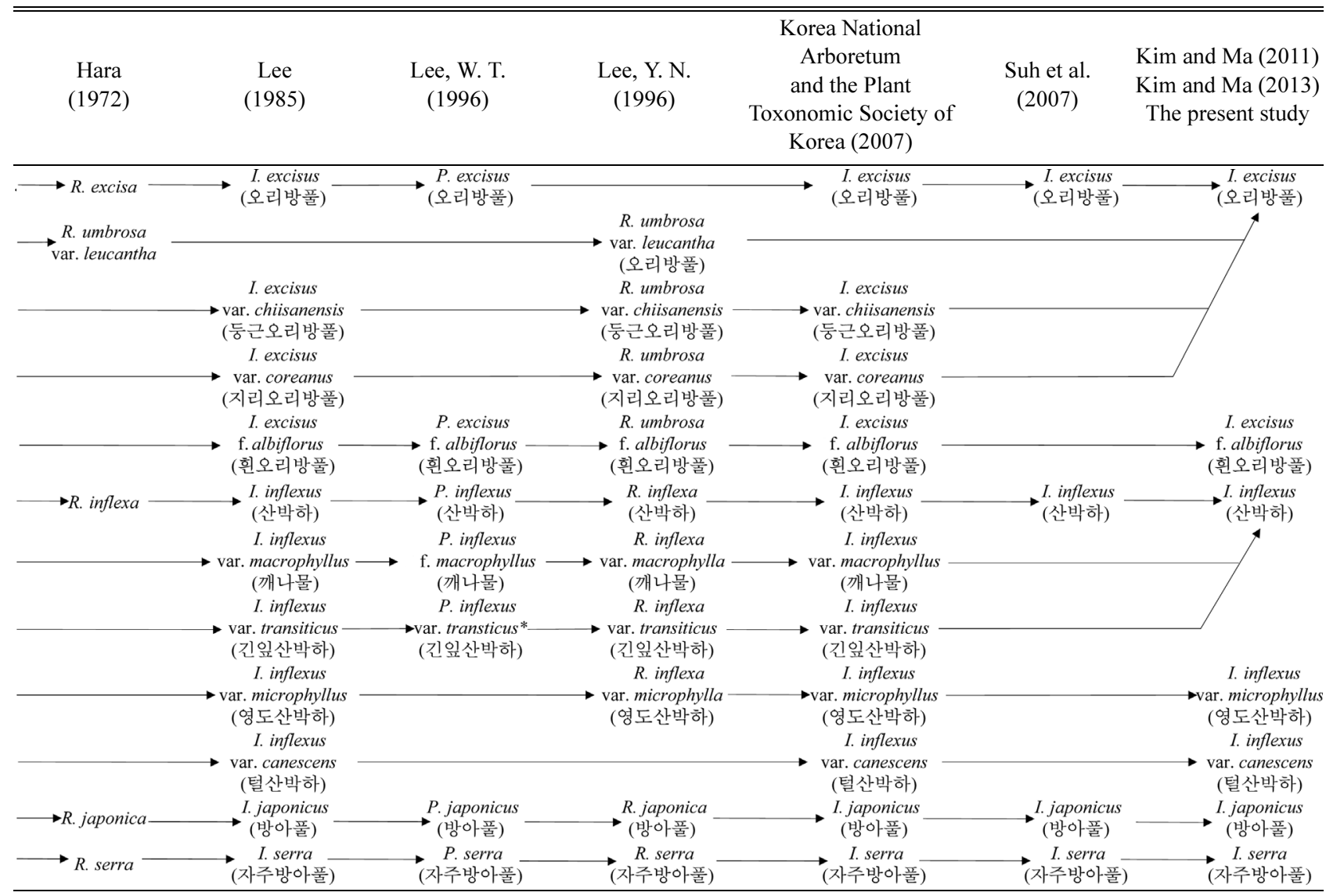

데 반하여 sect. Amethystoides는 종형 또는 넓은 종형의 꽃받 침과 성긴 원추화서를 갖고, sect. Isodon이 대부분 많은 꽃들 이 달린 취산화서를 갖고 꽃받침 잎은 각각 거의 동일한데 반하여 sect. Melissoides는 다소 적은 꽃들이 달린 취산화서 이고 꽃받침은 현저하게 두 부분으로 나누어져 있고 화통 상순 기부가 돌출한 거(spur)를 갖는 것이다.

최근 발표된 국가표준식물목록(Korean National Arboretum and the Plant Toxonomic Society of Korea, 2007)에서는 Li (1988)의 속 인식을 받아들여 Isodon 내의 분류군으로 $I$. excisus (오리방풀), I. excisus var. chiisanensis (둥근오리방 풀), I. excisus var. coreanus (지리오리방풀), I. excisus f. albiflorus (흰오리방풀), I. inflexus (산박하), I. inflexus var. macrophyllus (깨나물), I. inflexus var. transiticus (긴잎산박 하), I. inflexus var. microphyllus (영도산박하), I. inflexus var. canescens (털산박하), I. japonicus (방아풀), I. serra (자주방 아풀)의 4종 7 변종 1 품종을 기록하였고, The Genera of Vascular Plant of Korea (Suh et al., 2007)에서는 I. excisus, I. inflexus, I. japonicus, I. serra의 4종만을 인식하고 있다. 이 들 한국산 분류군들은 $\mathrm{Li}$ (1988)의 분류학적 재검토에 의 하면 종 수준에서 I. inflexus, I. japonicus, I. serra가 sect. Amethystoides에 속하고, I. excisus는 sect. Isodon에 속한다.

한반도에 분포하는 I. excisus와 I. inflexus의 종하 분류군
들의 구분에 대해서는 학자들마다 종 내 분류군 인식에 차이가 있어 왔는데(Table 1), 최근 이들에 대한 형태적 자 료의 주성분분석에 의한 연구는 백색품종인 I. excisus f. albiflorus를 제외하고 I. excisus의 종하 분류군들이 단일 분 류군으로 인식되어야 하며(Kim and Ma, 2011), I. inflexus는 세 개의 독립된 분류군인 var. inflexus, var. canescens, var. microphyllus로 인식되어야 함을 제시한 바 있다(Kim and $\mathrm{Ma}, 2013)$. 또한 한국산 Isodon 식물들에 대해서는 과피 및 화분의 표면형질에 대한 전자현미경적 조사가 이루어 진 바 있는데, 이 연구로 I. serra는 국내의 다른 분류군들 보 다 월등히 두꺼운 망벽의 화분을 갖고 과실의 표면에 비 선모만 고르게 분포하는 것이 다른 분류군들과 구분할 수 있는 특징으로 보고되었다(Ma and Kim, 2014).

본 연구에서는 최근 한국산 Isodon 식물들에 관계된 약 600점의 표본을 조사하여 이를 바탕으로 한국산 Isodon 식 물을 일곱 분류군(Table 1)으로 인식하였다. 이들의 외부 형태학적 형질을 재검토하고 각 분류군에 대한 형질들의 유형을 조사하여 분류군 간의 구분성을 파악하였고, 전자 현미경에 의해 줄기와 잎의 선모를 관찰하였으며, 본 연 구의 결과와 최근에 이루어진 연구들( $\mathrm{Kim}$ and $\mathrm{Ma}, 2011$, 2013; Ma and Kim 2014)를 종합하여 한국산 Isodon 식물에 대한 검색표를 작성하였다. 


\section{재료 및 방법}

본 연구에 사용된 재료는 성신여자대학교표본관(SWU),
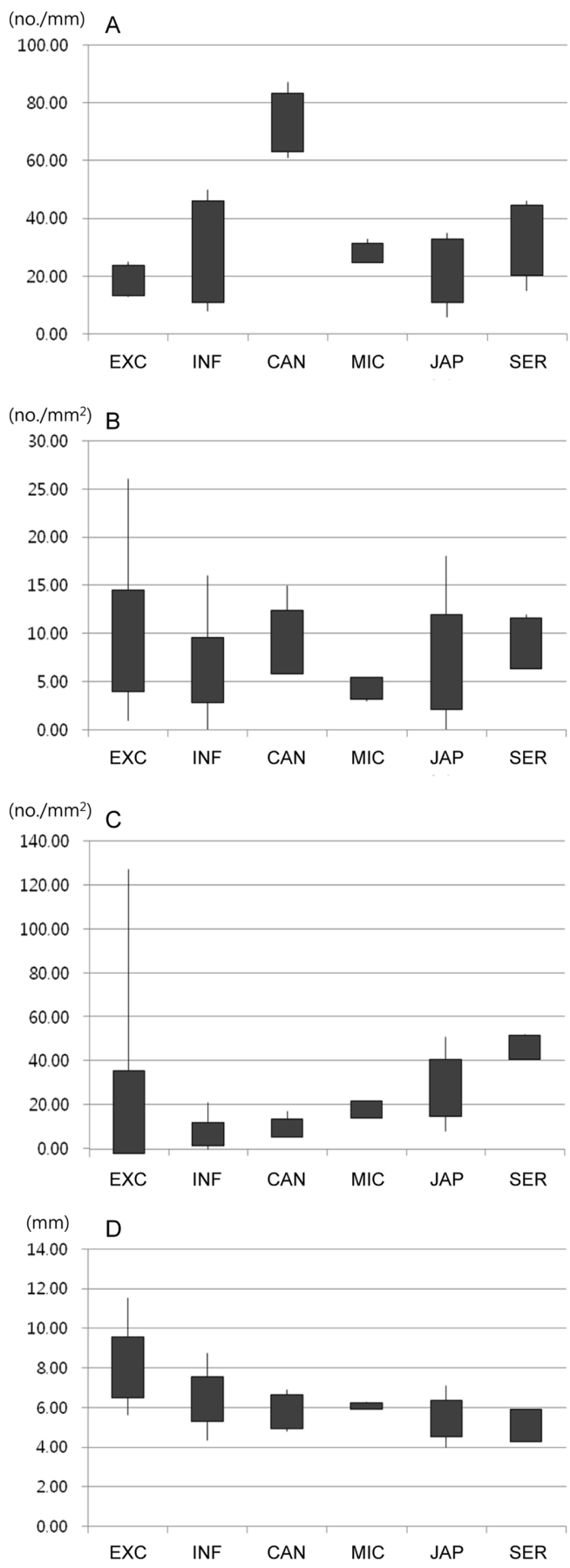

Fig. 1. A size-range diagram of the density of trichome in stem (\#/ $\mathrm{mm})(\mathrm{A})$, the density of glandular hairs in the adaxial (B) and abaxial (C) surfaces of the leaf $\left(\# / \mathrm{mm}^{2}\right)$, and the length of corolla (D). Squares indicate standard deviations and bars indicate maximum and minimum values. EXE, I. excisus; INF, I. inflexus var. inflexus; CAN, I. inflexus var. canescens; JAP, I. japonicus; MIC, I. inflexus var. microphyllus; SER, I. serra.
국립생물자원관표본관(NIBR), 국립수목원표본관(KH), 성 균관대학교표본관(SKK), 서울대학교표본관(SNU), 서울 대학교농업생명과학대학수목원표본관(SNUA), 고려대학 교표본관(KSU), 강원대학교표본관(KWNU), 충북대학교

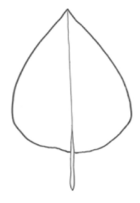

A

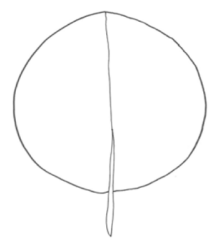

$\mathrm{E}$

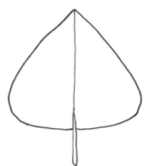

B

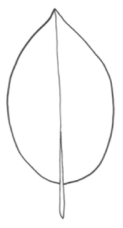

C

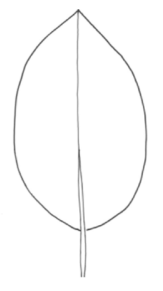

D

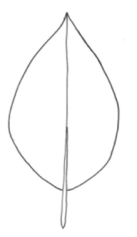

G
Fig. 2. Leaf shapes of the Korean Isodon. A. Ovate; B. Triangularovate; C. Elliptical; D. Oblong; E. Orbicular; F. Orbicular-ovate; G. Lanceolate-ovate.

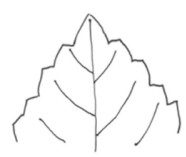

A

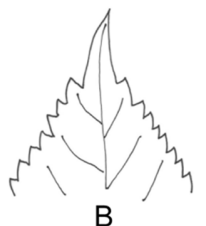

B

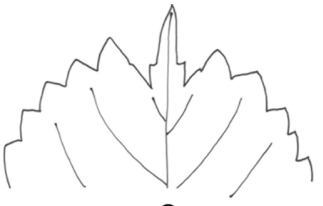

C
Fig. 3. Leaf apexs of the Korean Isodon. A. Acute; B. Acuminate; C. Excised and caudate.

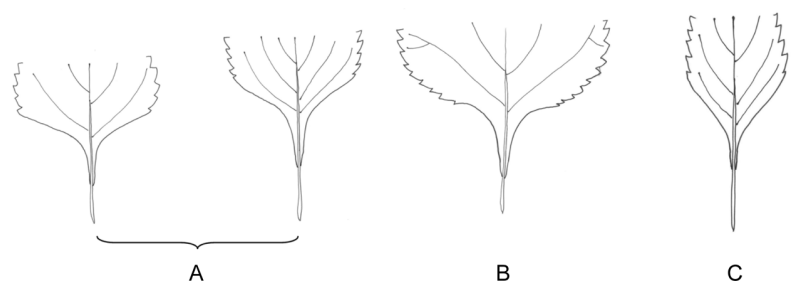

Fig. 4. Leaf bases of the Korean Isodon. A. Attenuate; B. Obtuse; C. Cuneate.

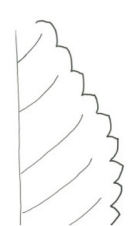

A

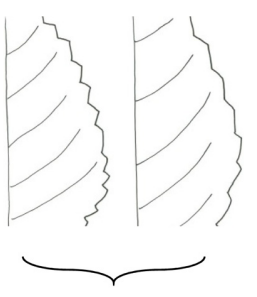

B

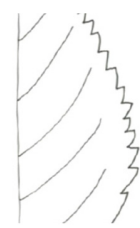

C

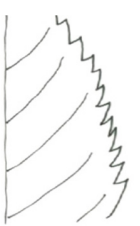

D
Fig. 5. Leaf margins of the Korean Isodon. A. Crenate; B. Dentate; C. Denticulate; D. Serrate. 
표본관(CBU), 일본 동경대학교표본관(TI)으로부터 대여 한 석엽표본 및 기준표본 슬라이드사진 약 600 점과 본 연 구자들이 직접 채집한 표본들을 포함하였다(Appendix 1). 자생지에서 채집된 식물은 포장에 재배하여 식물체의 성 장에 따른 형질의 변화를 관찰하였으며, 본 연구 과정에 서 직접 채집되어 제작된 석엽표본들은 성신여자대학교 표본관(SWU)에 소장하였다.

식물의 외부형태학적 형질에 대해서는 지금까지 발표 된 문헌들에서 이용된 형질들을 중심으로 취산화서의 형 태, 잎의 형태와 크기, 거치의 상태, 잎 표면의 선모와 비선 모의 분포 상태, 화서의 종류, 수술의 돌출여부, 소견과의 선모와 비선모의 여부 등에 대한 관찰 및 측정으로 이들 형 질들의 유형을 구분하였다. 양적인 형질들은 꽃이나 열매 가 있는 성숙한 개체를 선별하여 각각의 형질을 측정하였 는데, 식물체 엽신의 길이와 너비, 엽병의 길이, 거치의 간 격, 화관의 길이, 꽃받침의 길이와 너비, 소화경과 화경의 길이, 수술과 암술의 길이는 digital calipers (Mitutoyo, Japan)로 측정하였고, 잎의 엽선, 엽저와 거치의 각도는 각 도기로 측정하였다. 잎의 표면에 분포하는 선모와 비선모 의 밀도는 해부현미경(Leica, Swizerland)을 사용하여 관찰 및 측정하였다.

줄기와 잎 표면 선모의 존재는 I. serra를 구분하는 중요 한 특징으로 알려져 왔다(Lee, 1985). 이는 확대경 또는 해부 현미경에 의해 확인 가능하지만, 보다 자세한 관찰을 위해 주사전자현미경에 의해 조사하였다. 주사전자현미경에 의 해 관찰한 표본들은 Table 2에 별도로 정리하였다. 잎 표면
에 묻은 이물질을 제거하기 위하여 잎을 증류수가 담긴 초 음파세척기(NXP1002, Nexul, Korea)에서 약 20분 처리한 후 건조시킨 다음, 약 $30-40 \mathrm{~nm}$ 두께로 백금 이온을 증착시켜 주사전자현미경(JSM-7500F, Jeol, Japan)으로 관찰하였다.

\section{결 과}

\section{외부형태학적 형질관찰 및 유형구분}

뿌리: 한국산 Isodon에 속하는 분류군들의 뿌리는 분류 군별로 특징적 형태를 지니고 있지는 않았고, 분류군내에 서 생장 상태에 따른 변이만 관찰되었다. 모든 분류군에

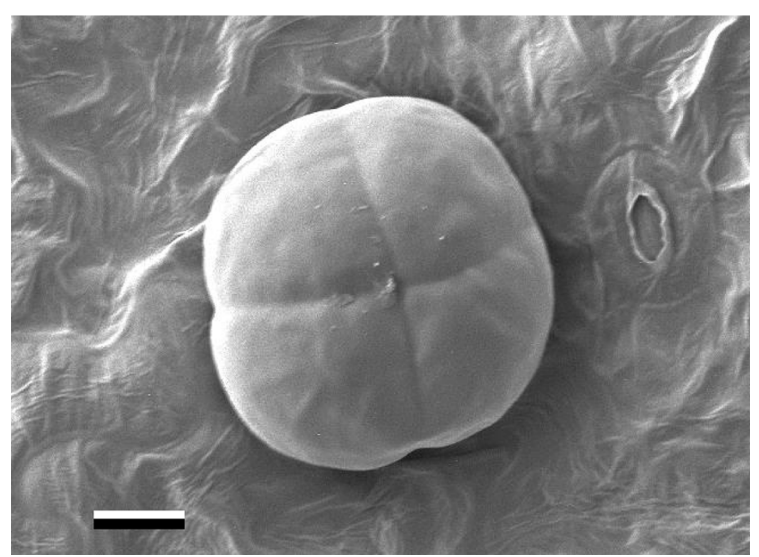

Fig. 6. A SEM picture of a glandular hair in the abaxial surface of $I$. serra. Scale bar is $10 \mathrm{um}$.

Table 3. A summary of leaf characters in the Korean Isodon.

\begin{tabular}{|c|c|c|c|c|c|c|}
\hline & I. excisus & $\begin{array}{l}\text { I. inflexus var. } \\
\text { inflexus }\end{array}$ & $\begin{array}{l}\text { I. inflexus var. } \\
\text { canescens }\end{array}$ & $\begin{array}{l}\text { I. inflexus var. } \\
\text { microphyllus }\end{array}$ & I. japonicus & I. serra \\
\hline \multicolumn{7}{|l|}{ leaf shape } \\
\hline ovate & - & + & - & + & - & - \\
\hline triangular-ovate & - & + & + & - & - & - \\
\hline elliptical & - & - & - & - & + & - \\
\hline oblong & - & - & - & - & + & - \\
\hline orbicular & + & - & - & - & - & - \\
\hline orbicular-ovate & + & - & - & - & - & - \\
\hline lanceolate-ovate & - & - & - & - & - & + \\
\hline \multicolumn{7}{|l|}{ leaf apex } \\
\hline acute & - & + & + & + & - & - \\
\hline acuminate & + & - & - & - & + & + \\
\hline excised and caudate & + & - & - & - & - & - \\
\hline \multicolumn{7}{|l|}{ leaf base } \\
\hline attenuate & - & + & + & + & + & - \\
\hline obtuse & + & - & - & - & - & - \\
\hline cuneate & - & - & - & - & - & + \\
\hline \multicolumn{7}{|l|}{ leaf margin } \\
\hline crenate & - & + & + & - & - & - \\
\hline dentate & + & + & - & + & - & - \\
\hline denticulate & - & - & - & - & + & - \\
\hline serrate & - & - & - & - & - & + \\
\hline
\end{tabular}

+, present; -, absent 
Table 4. Density of glandular hairs on the leaf surface of the Korean Isodon.

$\left(\right.$ no. $\left./ \mathrm{mm}^{2}\right)$

\begin{tabular}{ccccccc}
\hline \hline Taxa & I. excisus & $\begin{array}{c}\text { I. inflexus } \text { var. } \\
\text { inflexus }\end{array}$ & $\begin{array}{c}\text { I. inflexus } \text { var. } \\
\text { canescens }\end{array}$ & $\begin{array}{c}\text { I. inflexus } \text { var. } \\
\text { microphyllus }\end{array}$ & I. japonicus & I. serra \\
\hline $\begin{array}{l}\text { adaxial } \\
\text { surface }\end{array}$ & $9.26 \pm 5.29$ & $6.17 \pm 3.38$ & $9.13 \pm 3.27$ & $4.33 \pm 1.15$ & $7.06 \pm 4.90$ & $9.00 \pm 2.65$ \\
$\begin{array}{l}\text { abaxial } \\
\text { surface }\end{array}$ & $16.71 \pm 19.04$ & $6.85 \pm 5.31$ & $9.50 \pm 4.11$ & $18.00 \pm 4.00$ & $27.61 \pm 13.05$ & $46.33 \pm 5.51$ \\
\hline
\end{tabular}

서 1-2년생으로 뿌리의 발달이 미약한 개체들은 잔뿌리만 주위로 짧게 뻗어 있고, 오랜 기간 생존한 개체들의 뿌리 는 타원형 또는 불규칙하게 비후되어 목질화 된 부분으로 부터 잔뿌리들이 나와 있다.

식물체의 높이: 식물체의 높이는 생장상태와 양지/음지 등의 생육환경에 따라 다르며, 성숙한 개체는 $30 ~ 150 \mathrm{~cm}$ 로 다양하다. 대부분의 분류군들이 생장초기에는 직립하는 데, 줄기가 더 길어질수록 사선으로 생장하여 식물체의 정 확한 높이를 측정하기는 어렵다. I. inflexus var. microphyllus 는 특징적으로 성숙한 개체가 높이 $30-50 \mathrm{~cm}$ 로 사선으로 자라고 많은 가지를 쳐서 다른 분류군과 구분되지만, 그 이외에 식물체의 높이는 한국산 Isodon 식물을 구별하는 데 유용하지 못한 형질이다.

줄기 단면 및 비선모의 밀도: 줄기의 단면은 능선이 뚜 렷한 능각형(ribbed square)과 능선이 거의 발달하지 않는 둔 각형(rounded square)으로 구분할 수 있지만, 모든 분류군의 어린 가지에서는 능각형이고 성장하면서 둔각형이 되어 식 물체의 아랫부분에서는 원형에 가깝게 된다. 그러므로 분 류군별로 줄기의 단면형을 나누기는 어렵다. 줄기의 비선 모들의 방향은 대부분 아래로 향하나 방향성 없는 것도 많 이 발견되는 등 개체 변이가 심하여 이 형질 또한 분류군을 구분할 수 있는 형질이 되지 못한다. 그러나 비선모의 밀도 는 I. inflexus var. canescens에서 평균 73 개/mm (줄기의 한쪽 면에서 보이는 비선모) 이상의 높은 밀도를 갖는 반면 다른 모든 분류군들은 약 $18-32$ 개 $/ \mathrm{mm}$ 의 밀도를 나타낸다(Fig. 1)

엽형: 잎의 형태는 크게 일곱 가지의 유형으로 구분되 지만 각 분류군마다 다양한 잎의 변이가 존재하여 각각의 분류군은 대부분 연속된 여러 가지 유형을 갖는다(Fig. 2; Table 2). 난형(ovate)을 갖는 식물은 I. inflexus var. inflexus, I. inflexus var. microphyllus, 삼각상난형(triangular-ovate)은 $I$. inflexus var. inflexus, I. inflexus var. canescens, 타원형 (elliptical)과 장타원형(oblong)은 I. japonicus, 원형(orbicular) 과 난상원형(orbicular-ovate)은 I. excisus, 난상피침형 (lanceolate-ovate)은 I. serra에서 발견된다. I. inflexus var. canescens와 I. inflexus var. microphyllus는 관찰된 표본 모두 에 있어서 각각 삼각상난형과 난형의 엽형이 관찰되었다.

엽두: 엽두의 형태는 크게 세 가지 유형으로 구분되는데 (Fig. 3; Table 2), 예두(acute)는 I. inflexus의 세 변종에서, 점 첨두(acuminate)는 I. excisus, I. japonicus, I. serra에서, 엽두 가 찟어진 꼬리모양(excised and caudate)은 I. excisus 에서 발견된다. I. inflexus의 세 변종과 I. japonicus, I. serra는 엽형
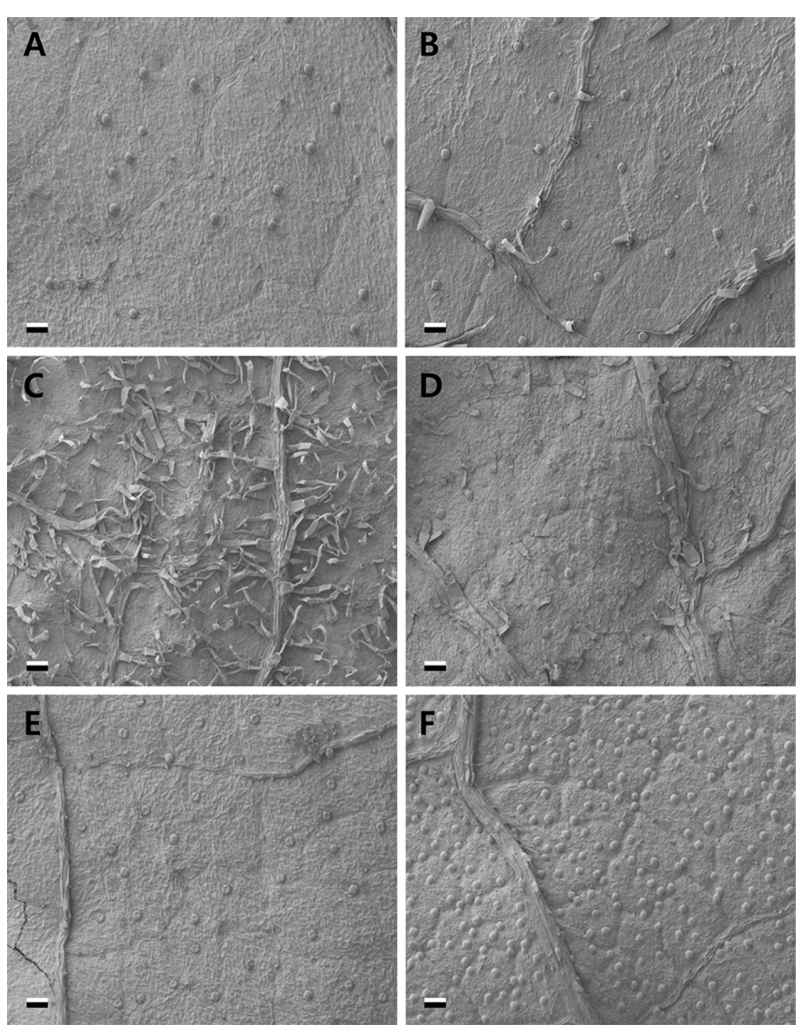

Fig. 7. SEM pictures of abaxial leaf surfaces of Korean Isodon. A. I. excisus; B. I. inflexus var. inflexus; C. I. inflexus var. canescens; D. I. inflexus var. microphyllus; E. I. japonicus; F. I. serra. Scale bar is $100 \mathrm{um}$.

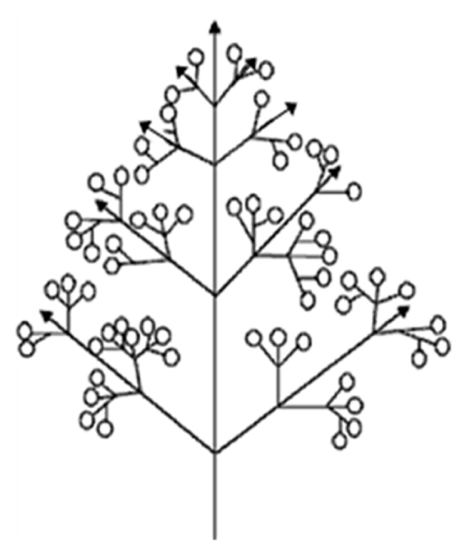

A

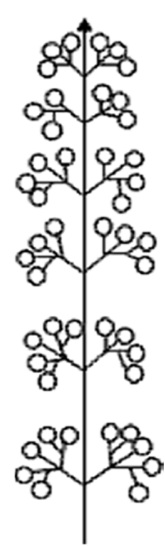

B
Fig. 8. Inflorescences of the Korean Isodon. A. Panicle; B. Therse. 


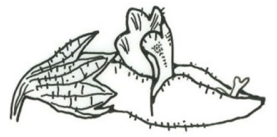

A

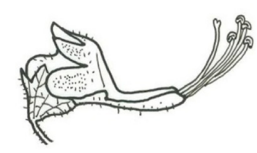

B

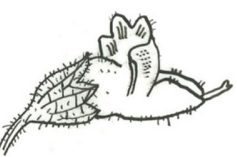

C
Fig. 9. Corollas of the Korean Isodon. A. Inflexus type; B. Japonicus type; C. Serra type.

의 변이는 다양하지만 엽두의 형태는 비교적 일정하며 각 각 예두(I. inflexus)와 점첨두(I. japonicus, I. serra)를 갖는다. I. excisus에서는 드믈게 점첨두와 찢어진 꼬리모양의 중간 형태를 보이는 개체가 발견되기는 하지만 난상 또는 난상 원형의 엽형의 특징을 동시에 고려하면 I. excisus를 다른 분류군으로부터 구분할 수 있다.

엽저: 엽저는 세 가지 유형으로 구분되는데(Fig. 4; Table 2), 유저(attenuate) 는 I. inflexus와 I. japonicus에서 관찰되며, 둔저(obtuse)는 I. excisus에서, 설저(cuneate)는 I. serra에서 관찰된다. 난형에서 삼각상난형인 잎의 형태를 갖는 $I$. inflexus는 넓은 엽선을 유지하다가 급격히 좁아지는 유저 를 갖으며, I. japonicus는 I. inflexus에 비하여 다소 완만한 유 저를 갖는다. I. serra는 잎 밑이 쐐기모양으로 기부를 향해 점점 뾰족해지는 설저를 갖고, I. excisus는 늘어진 둔저를 갖는데, 간혹 평저에 가까운 둔저를 갖는 개체도 관찰된다.

엽연: 엽연은 네 가지 유형으로 구분는데(Fig. 5; Table 2), 둔거치(crenate)는 I. inflexus var. inflexus와 I. inflexus var. canescens에서, 치상거치(dentate)는 I. inflexus var. inflexus, I. inflexus var. microphyllus, I. excisus에서, 소치상거치(denticulate)는 I. japonicus에서, 예거치(serrate)는 I. japonicus와 $I$. $s e r r a$ 에서 발견된다. 거치의 모양 또한 한 분류군 내에서도 여러 가지 유형이 연속적으로 나타나는 경우가 관찰되는 데, I. inflexus var. inflexus, I. inflexus var. microphyllus 는 주로 치상거치를 보이지만, I. inflexus var. inflexus와 I. inflexus var. canescens는 간혹 둔거치를 보인다. 이들 분류군에서는 한 개체에서 치상거치와 둔거치를 함께 가지는 개체도 관찰 된다. I. excisus는 특히 거치가 다양하게 관찰되는데, 대부 분의 개체가 치상거치를 보이나 높이가 낮은 치상거치를 보이기도 한다. 간혹 엽두의 찢어진 꼬리에 1-2개의 예거 치를 갖기도 한다.

잎 표면의 선모: 기존에 발표된 우리나라 대부분의 도감 및 검색표에서는 잎 뒷면의 선모의 존재가 I. serra를 한국 산 Isodon의 다른 분류군들과 구분할 수 있는 형질로 제시 되어 있었지만(Lee, 1985; Lee, W. C., 1996; Lee, Y. N., 1996; Lee, 1997), 본 연구에서는 관찰한 모든 한국산 Isodon 식물 들의 잎 앞 - 뒷면에 선모를 갖고 있음을 관찰하였다(Fig. 6). 선모밀도에 대한 측정결과 일반적으로 잎 앞면에서는 뒷면보다는 적은 선모를 갖고 있었는데, 앞면의 선모는 분류군 간의 차이는 거의 없었고, 뒷면은 $I . s e r r a$ 가 $\mathrm{mm}^{2}$ 당 평균 46.33 개의 월등히 많은 선모를 갖고 있어 다른 분류 군과 구분되었다(Fig. 1 and 7, Table 4). I. excisus와 I. japonicus의 잎 뒷면의 선모는 각각 $\mathrm{mm}^{2}$ 당 평균 16.71 개, 27.61개의 선모를 갖고 있었지만 매우 큰 변이 폭을 갖고 있다(Fig. 1, Table 4).

화서: Isodon에 속하는 분류군의 화서는 무한화서인 원 추화서(panicle)와 복합화서인 밀추화서(therse)가 관찰된 다(Fig. 8). 원추화서를 갖는 분류군은 I. japonicus, I. serra, 밀추화서를 갖는 분류군은 I. excisus와 I. inflexus에 속하는 분류군들이 여기에 속한다.

꽃: 화관은 순형으로 상순과 하순으로 나누어지는데, 상 순 끝은 위로 굽어 4 개로 갈라지고, 하순은 밑으로 볼록하 게 쳐져 굽는다. 화관의 색깔은 I. excisus f. excisus와 I. inflexus 의 세 변종들, I. serra는 보라색을, I. japonicus는 흰색이 도는 연보라색을 띠며 모든 분류군에서 갈라진 상순 위로 진한 보라색의 점들이 무늬를 이룬다. 완전히 백색의 화관을 갖 는 분류군은 I. excisus f. albiflorus이다. 화관의 평균 크기는 I. excisus 가 $8.03 \mathrm{~mm}$, I. inflexus var. inflexus 가 $6.43 \mathrm{~mm}$, I. inflexus var. microphyllus 가 $6.08 \mathrm{~mm}$, I. inflexus var. canescens 가 $5.8 \mathrm{~mm}$, I. japonicus 가 $5.49 \mathrm{~mm}$, I. serra가 $4.95 \mathrm{~mm}$ 순으로 그 변이 폭 이 대부분 겹치지만 I. excisus와 I. serra는 서로 변이폭이 겹 치지 않고 구분된다(Fig. 1). 화관의 형태는 세 가지 유형으 로 구분되는데(Fig. 9), 산박하형(INFLEXUS type)은 화통이 길고, 암 - 수술이 하순에 거의 모두 싸여있는 형태로 $I$. excisus와 I. inflexus의 세 변종이 여기에 속한다. 방아풀형 (JAPONICUS type)은 화통이 짧고, 암 - 수술이 하순길이 이 상으로 돌출되어 있는 형태로 I. japonicus가 여기에 속한다. 자주방아풀형(SERRA type)은 화통이 짧고, 수술은 하순에 싸여있으나 암술은 하순보다 길어 하순 밖으로 돌출되어 있는 형태로 I. serra가 여기에 속한다. 2강웅예인 수술은 $I$. inflexus의 세 변종과 I, excisus, I. serra에 있어서는 큰 2 개의 수술이 암술보다 약간 짧고 하순에 싸여있지만, I. japonicus 에 있어서는 암술과 거의 비슷한 크기로 하순 끝으로부터 하순길이 이상으로 더욱 돌출되어 있다.

\section{고 찰}

Isodon의 국명은 여러 문헌에 있어서 “산박하속”(Park, 1974; Lee, 2006; Suh et al., 2007)과 “방아풀속”(Lee, 1997)으 로 서로 달리 사용되어 왔다. 종내 가장 먼저 발표된 종의 종명을 채택해 속명을 사용한다면 한국산 Isodon 분류군 들 중에는 방아풀로 국명이 사용되는 Scutellaria japonica $(=I$. japonica)가 가장 먼저 보고되었으므로(Burmann, 1768) Isodon은 “방하풀속”을 사용되어야 할 것이다. 반면 우리나라 식물들을 종합하여 정리한 도감, 명집 중 가장 먼저 속에 대한 국명을 사용한 것을 채택하여야 한다면 한국쌍자엽식물지(Park, 1974)에서 가장 먼저 속의 국명 을 “산박하속”이라 사용했음으로 이를 사용해야 할 것이 다. 최근 우리나라 식물의 이름이 정리된 국가표준식물목 록(Korea National Arboretum and the Plant Toxonomic 
Society of Korea, 2007)에서는 “산박하속"을 사용하였고 본 연구는 후자의 견해를 따랐다.

본 연구에서는 한국산 Isodon 분류군들에 대하여 최근 이 루어진 다변량분석에 의한 분류군 인식(Kim and $\mathrm{Ma}, 2011$, 2013) 및 과피와 화분의 표면형질에 대한 연구(Ma and Kim, 2014)와 함께 본 연구에서 이루어진 외부형태적 형질 관찰, 주사전자현미경을 통한 모용 관찰 등을 종합하여 일곱 개 의 분류군을 인식하였고, 이들을 구분할 수 있는 형질들을 조사하였다. 이들 분류군들 중 Nakai (1934)에 의해서 인식 된 바 있는 I. excisus의 두 변종인 var. coreanus와 var. chiisanensis 는 지금까지 대부분의 문헌에서 그 국명이 각각 지리오리방풀과 둥근오리방풀로 사용되어 왔는데(Lee, 1985; Lee, Y. N., 1996; Korean National Arboretum and the Taxonomic Society of Korea, 2007; Suh et al., 2007), 원기재의 검토에 의하면 var. chiisanensis는 지리산에서 발견된 것이고, var. coreanus는 잎이 둥글다고 기록되어 있어 Lee (1985)에 의해 이들 두 분류군들에 대한 국명이 바뀌어 부여된 이래 지금까지 이들 이름이 지속적으로 사용되어 왔던 것으로 생각된다. 또한 Lee (1969)가 발표한 이들 분류군의 기본명 에 대한 새로운 조합명인 I. excisus var. chiisanensis와 I. excisus var. coreanus는 기본명에 대한 문헌을 제공하지 않아 국제조류균류식물명명규약(Melbourne Code, 2011; Article 41.3)에 의해 비합법명이다(Kim et al., 2005). 그러나 최근의 외부형태학적 형질에 의한 주성분 분석 연구 결과는 종내 분류군들 간의 구분을 지지해주지 못하여 이들을 단일 분 류군으로 인식해야 함을 제시한 바 있어(Kim and Ma, 2011), 이들에 대한 학명 및 국명의 정확한 사용에 대한 논의는 무 의미하다.

I. inflexus의 변종들은 원기재에서 I. inflexus var. inflexus 중 잎의 크기가 큰 분류군을 I. inflexus var. macrophyllus로, 긴 잎 을 갖는 분류군을 I. inflexus var. transiticus로 인식하였는데, 최근의 외부형태학적 형질의 주성분분석 결과는 두 분류 군들 모두 I. inflexus var. inflexus와 동일 분류군으로 인식해 야 함을 지지하고 있다(Kim and Ma, 2013). I. inflexus var. inflexus에 해당하는 분류군에서 매우 드물게 엽두의 형태가 I. excisus에서 나타나는 꼬리모양을 갖는 개체가 관찰되지 만, I. excisus는 원형 또는 난상원형의 엽형과 평균 $8 \mathrm{~mm}$ 길 이의 화관을 갖고, I. inflexus는 난형 또는 삼각상 난형의 엽 형과 평균 $6.5 \mathrm{~mm}$ 길이의 화관을 가져 이들 분류군들은 위 의 형질들의 조합으로 식별 가능하다. Lee Y. N. (1996)는 $R$. inflexa f. albiflora (흰산박하)를 새로운 품종으로 발표하였지 만 기준표본을 제시하지 않아 국제조류균류식물명명규약 (Melbo-urne Code, 2011; Article 6)에 의해 비합법명이다. 본 연 구에서는 I. inflexus 내에서 관찰된 흰색 꽃의 표본을 발견하 지 못하여 흰산박하에 대한 분류학적 판단은 보류하였다.

I. inflexus var. microphyllus는 I. inflexus 내의 분류군들에 대 한 외부형태학적 형질의 주성분분석에서 I. inflexus 내의 다른 분류군들과 확연히 구분됨이 확인되었는데(Kim and
$\mathrm{Ma}, 2013)$, 주사전자현미경에 의한 과피 표면의 연구에서 도 비선모가 과실 전체에 분포한다는 점이 한국산 Isodon 에 속하는 다른 모든 분류군들과 확연히 구분됨을 보여주 었다(Ma and Kim, 2014). I. inflexus var. microphyllus는 한국 산 Isodon에 속하는 다른 모든 분류군과 확연히 구분되는 형질들이 다수 발견되는 것은 이 분류군의 독립된 종으로 서의 위치를 지지해주는 결과이지만, 본 연구에 있어서 관 찰된 표본이 3점에 불과해 앞으로 더 많은 표본을 추가한 연구에 의한 검토가 필요하다. I. inflexus var. canescens는 외 부형태학적으로 화관의 형태와 화서의 형태가 I. inflexus var. inflexus와 비슷하나, 엽형이 뚜렷한 삼각상난형이고 줄 기 전체와 잎의 표면에 수많은 비선모들이 분포한다는 점 이 I. inflexus var. inflexus와는 다르다.

I. japonicus는 한국산 Isodon의 다른 분류군들과는 암 - 수 술이 모두 화관 밖으로 돌출되어 있어 쉽게 구별된다(Fig. 7B). 그러나 잎의 크기나 형태의 변이가 다양하여 잎의 형질 만으로는 다른 분류군들과 쉽게 구분되지 않고, I. excisus와 I. inflexus와는 다르게 원추화서를 형성하고 있다. I. serra는 비슷한 분류군인 I. japonicus와 화관의 형태(Fig. 9 C) 이외에 도 소견과는 정단에만 짧은 비선모를 갖고 있어 확연하게 구별된다(Ma and Kim, 2014). 또한 잎 뒷면에 선모가 현저하 게 많고(Fig. 1 and 7, Table 4), 개화기는 9-10월로 늦어 한국산 Isodon의 다른 분류군들과 뚜렷하게 구별된다. I. japonicus와 I. serra의 국명은 각각 방아풀과 자주방아풀로 두 분류군 간 의 가까운 연관성을 내포한 국명으로 사용되어 왔지만 (Chung, 1957; Lee, 1985; Lee, W. T., 1996; Lee, Y. N., 1996), 이 들은 뚜렷이 구분되는 독립적인 종들이다.

위의 결과와 논의를 바탕으로 한국산 Isodon은 4 종 2 변종 1 품종으로 정리되었고, 이를 바탕으로 검색표가 작성되었다. 최근 연구된 과피표면형질(Ma and Kim, 2014)은 현장에서 확대경에 의해 확인할 수 있는 형질 이므로 이를 검색표에 포함시켰다.

\section{한국산 Isodon (산박하속) 분류군 검색표}

1. 잎은 원형에서 난상원형이고 엽두가 꼬리모양이다

2. 화피의 색이 보라색이다 .. 오리방풀 I. excisus f. excisus

2. 화피의 색이 흰색이다

흰오리방풀 I. excisus f. albiflorus

1. 잎은 난형, 삼각상난형, 타원형 또는 피침형이고 엽 두가 예두에서 점첨두이다

3. 화서는 밀추화서이고, 화경은 화관보다 짧다

4. 줄기의 한쪽 면에서 관찰되는 비선모는 $1 \mathrm{~mm}$ 당 70 개 이상이다

털산박하 I. inflexus var. canescans

4. 줄기의 한쪽 면에서 관찰되는 비선모는 $1 \mathrm{~mm}$ 당 40 개 이하이다

5. 잎은 길이 $0.6 \sim 2 \mathrm{~cm}$ 너비 $0.3 \sim 1.5 \mathrm{~cm}$ 정도이고, 잎 
뒷면의 비선모는 약 5 개 $/ \mathrm{mm}^{2}$ 로 다소 성기다

영도산박하 I. inflexus var. microphyllus

5. 잎은 길이 $3 \sim 15 \mathrm{~cm}$ 너비 $2 \sim 8,5 \mathrm{~cm}$ 정도이고, 뒷면 의 비선모는 약 18 개 $/ \mathrm{mm}^{2}$ 로 다소 밀하다 산박하 I. inflexus var. inflexus

3. 화서는 원추화서이고, 화경은 화관보다 길다

6. 암술과 수술이 화관의 하순 길이 이상으로 하순 밖 으로 돌출됨. 화관은 연한 보라색 이고 잎 뒷면에 40 개 $/ \mathrm{mm}^{2}$ 이하(평균 28 개 $/ \mathrm{mm}^{2}$ )의 선모가 있으며, 소견과 정단에 선모만 분포하고, 비선모는 분포하 지 않는다 …………………………방아풀 I. japonicus

6. 수술이 화관의 하순에 싸여있고 암술만 하순으로 부터 약간 돌출됨. 화관은 보라색이고 잎 뒷면에 40 개 $/ \mathrm{mm}^{2}$ 이상 (평균 46 개/ $/ \mathrm{mm}^{2}$ )의 선모가 있으며, 정단을 포함한 소견과 전체에 선모와 비선모가 모 두 분포한다 자주방아풀 I. serra

\section{사 사}

본 논문은 2014년도 성신여자대학교 학술연구조성비 지원에 의하여 연구되었음을 밝힙니다.

\section{인용문헌}

Bentham, G 1836. Labiatarum Genera et Species. James Ridgway and Sons, London.

Burmann, F. 1768. Flora Indica cui Accedit Series Zoophytorum Indicorum nec non Prodromus Florae Capensis. Apud Johannem Schreuderum, Amstelaedami.

Chung, T. H. 1957. Korean Flora. Kyo-yuk publishing Co. Seoul. (in Korean)

Halrey, R. M., S. Atkins, A. L. Budantsev, P. D. Cantino, B. J. Conn, R. Grayer, M. M. Harley, R. De Kok, T. Krestovskaja, R. Morales, A. Paton, O. Ryding, and T. Upson. 2004. Labiatae. In The Families and Genera of Vascular Plant, Vol. VII. Flowering Plants: Dicotyledons (Lamiales Except Acanthaceae Including Avicenniaceae). Kadereit, J. W. (ed.), Springer Verlag, Berlin and Heidelberg.

Hara, H. 1972. On the asiatic species of the genus Rabdosia (Labiatae). Journal of Japanese Botany 47: 193-203.

Kim, H., H. S., Lee, S. Park, and C. S. Chang. 2005. Invalid names published by T. B. Lee. Korean Journal of Plant Taxonomy 35: 211-226. (in Korean)

Kim, S. and Y. Ma. 2011. Morphological multivariate analyses of Isodon excisus complex (Lamiaceae) in Korea. Korean Journal of Plant Taxonomy 41: 223-229.

Kim, S. and Y. Ma. 2013. Morphological multivariate analyses of Isodon inflexus complex (Lamiaceae) in Korea. Korean Jour- nal of Nature Conservation 7: 42-49. (in Korean)

Koidzumi, G. 1929. Contributiones ad cognitionem florae asiae orientalis. Botanical Magazine Tokyo 43: 386.

Korea National Arboretum and the Plant Toxonomic Society of Korea. 2007. A Synonymic List of Vascular Plants in Korea. Korea National Arboretum, Pocheon.

Kudo, Y. 1929. Memories of the Faculty of Science and Agriculture. Vol II. No. 2. Taihoku Imperial University, Formosa.

Lee, T. B. 1985. Illustrated Flora of Korea. Hyang-mun publishing Co. Seoul. (in Korean)

Lee, T. B. 2006. Illustrated Flora of Korea. $2^{\text {nd }}$ ed. Hyang-mun publishing Co. Seoul. (in Korean)

Lee, W. T. 1996. Colored Standard Illustrations of Korean Plants. Academy publishing Co., Seoul. (in Korean)

Lee, Y. N 1996. Flora of Korea. Kyo-hak Publishing Co. Seoul. (in Korean)

Lee, S. 1997. Key to the Korean Plants. Academy publishing Co., Seoul. (in Korean)

Li, H. 1988. Taxonomic review of Isodon (Labiatae). Journal of the Arnold Arboretum 69: 289-395.

Ma, Y. and S. Kim. 2014. Pollen and fruit morphology of Isodon (Lamiaceae) in Korea. Korean Journal of Nature Conservation. In press (in Korean)

Mabberley, D. J. 1987. The Plant-Book: A Portable Dictionary of the Higher Plants. Cambridge University Press, New York.

Maki, M., T. Yamashiro, I., Dohzono, and K. Suzuki. 2010. Molecular phylogeny of Isodon (Lamiaceae) in Japan using chloroplast DNA sequences: recent rapid radiations or ancient introgressive hybridization? Plant Speciation Biology 25: 240-248.

Maximowicz, C. J. 1875. Mélanges Biologiques Tires du Bulletin Physico-Mathematique de l'Academie Imperiale des Sciences de St.-Pétersbourg. St. Petersburg, Leipzig.

Mori, T. 1921. An enumeration of plants hitherto known from Korea. The government of Chosen, Seoul.

Nakai, T. 1911. Flora Koreana. Journal of the college of science, Imperial University of Tokyo 31: 1-573.

Nakai, T. 1921. Labiatae coreanae. Botanical Magazine Tokyo 35: 191-205.

Nakai, T. 1934. Notulae ad plants Japoniae \& Koreae. Botanical Magazine Tokyo 48: 785-792.

Park, M. 1974. Keys to the Herbacious Plants in Korea. JungeumSa, Seou. (in Korean)

Suh, Y., S. Hong, and S. Park. 2007. Lamiaceae. In The Genera of Vascular Plants of Korea. Flora of Korea Editorial Committee ed. Academy, Seoul.

Zhong, J.-S., J. Li, L. Li, J. G. Conran, and H. Li. 2010. Phylogeny of Isodon (Schrad. ex Benth.) Spach (Lamiaceae) and related genera inferred from nuclear ribosomal ITS, trnL-trnF region, and rps 16 intron sequences and morphology. American Society of Plant Taxonomists 35: 207-219. 


\section{APPENDIX 1}

\section{Isodon excisus (Maxim.) Kudo f. excisus 오리방풀}

Gangwon-do: Daegwanryeong Daegwanryeong-myeon Pyeongchang-gun, Aug. 13 2006, E.-S. Jeon ESJeon61160 (KH); Daegwanryeong Daegwanryeong-myeon Pyeongchang-gun, Aug. 20-21 1969, T. Lee s. n. (SNUA); Dakmongnyeong Wangsan-myeon Gangneung-si, Jul. 27 2007, H.-G. Oh et al. Daegwallyeong-0087 (KH); Dong River Yeongwol-eup Yeongwol-gun, Sep. 3 2005, Hong et al. KHUS0312 (KH); Duwibong Jeongseon-gun, Jul. 3 2008, S.-H. Oh et al. s. n. (KH); Galjeongokbong Girin-myeon Injegun, Sep. 19 2008, K. Lee et al. Gj-0800276 (KH); Gwangdeok-ri Sanae-myeon Hwacheon-gun, Sep. 30 2006, E.-S. Jeon ESJeon1777 (KH); Hwaamsa Sinpyeong-ri Toseong-myeon Goseong-gun, Sep. 13 2002, H. Kim et al. s. $n$. (NIBR); Jindong-ri Girin-myeon Injegun, Aug. 14 1997, C. Chang et al. JB245 (NIBR); Jochimryeong Seo-myeon Yangyang-gun, Aug. 1 2007, H.-W. Kim s. n. (KSU); Jochimryeong Seo-myeon Yangyang-gun, Sep. 1 2007, H.-W. Kim S. n. (KSU); Misiryeong Yongdae-ri Buk-myeon Inje-gun, Sep. 7 2008, H.-W. Kim et al. s. n. (KSU) two sheets; Mt. Baegun Panbu-myeon Wonju-si, Aug. 17 2003, H.-J. Bang et al. s. n. (KSU); Mt. Baegun Panbu-myeon Wonju-si, Aug. 17 2003, K.-J. Kim et al. s. n. (KSU) three sheets; Mt. Baegun Panbu-myeon Wonju-si, Aug. 5 1993, H. Won 93-7-73 (SNU); Mt. Baekdeok Suju-myeon Yeongwol-gun, Sep. 14 2001, D. Choi et al. s. n. (NIBR); Mt. Baekjeokm Pyeongchang-gun, Sep. 5 2008, J.-C. Yang Baekjeoksan80905-9 (KH); Mt. Bangtae Bangdong-ri Girin-myeon Inje-gun, Aug. 16 2008, W. Paik NAPI-0360, NAPI-0361, NAPI-0362 (KH); Mt. Bangtae Bangdong-ri Girin-myeon Inje-gun, Jul. 292008, K. Lee et al. BT-0800235 (KH); Mt. Barwang Daegwanryeong-myeon Pyeongchang-gun, Oct. 8 1978, B.-Y. Sun s. n. (SNU); Mt. Cheolgapryeong Yeonok-myeon Gangneung-si, Jul. 24 1998, H.-W. Lee et al. s. n. (SNU); Mt. Chiak Socho-myeon Wonju-si, Sep. 21 1996, E. Cho s. n. (SNU); Mt. Daeam Inje-gun, Sep. 27 2006, J.-S. Kim et al. s. n. (KSU); Mt. Daeam Inje-gun, Sep. 272006 , J.-S. Kim et al. s. n. (KSU); Mt. Daeam Seoheung-ri Seohwa-myeon Inje-gun, Aug. 15 2008, W. Paik NAPI-0267 (KH); Mt. Daeam Seoheung-ri Seohwa-myeon Inje-gun, Sep. 19 2008, W. Paik NAPI-0349, NAPI-0265 (KH); Mt. Daeam Seoheung-ri Seohwa-myeon Inje-gun, Sep. 19 2008, W. Paik s. n. (KH) two sheets; Mt. Deokhang Singi-myeon Samcheok-si, Aug. 6 2005, S. Lee et al. s. n. (KH); Mt. Dosol Yanggu-gun, Jul. 11 2003, E.-S. Jeon ESJeon31082 (KH); Mt. Duta Samcheok-si, Jul. 28 2007, J. Hyun et al. 902024 (KH); Mt. Duta Samcheok-si, Sep. 8 2007, J. Hyun et al. s. n. (KH); Mt. Gari Duchon-myeon Hongcheon-gun, Sep. 18 1976, Y. Kil s. n. (KWNU); Mt. Gariwang Jeongseon-eup Jeongseon-gun, Aug. 9 1990, Y. Jung et al. s. n. (SKK); Mt. Gwangdeok Sanaemyeon Hwacheon-gun, Jul. 6 2009, M. Kong et al. s. n. (KH); Mt. Gwangdoeg Hwacheon-gun, Sep. 5 2003, E. Jung et al. K0905022 (KH); Mt. Gyebang Jinbu-myeon Pyeongchang-gun, Aug. 23 2005, K.-J. Kim et al. s. n. (KSU) two sheets; Mt. Gyebang Jinbumyeon Pyeongchang-gun, Jul. 26 2003, K. Heo et al. s. n. (SKK); Mt. Jeombong Inje-eup Inje-gun, Aug. 11 2005, S. Lee SKK1059 (KH); Mt. Jeombong Inje-eup Inje-gun, Sep. 28 2006, J.-S. Kim et al. s. n. (KSU); Mt. Jeombong Seorak-dong Sokcho-si, Jul. 30 2008, W. Paik NAPI-0336 (KH); Mt. Jeombong Seorak-dong Sokcho-si, Jul. 9 2008, W. Paik NAPI-0257 (KH); Mt. Ma Toseongmyeon Goseong-gun, May 17 2008, W. Paik NAPI-0328 (KH) two sheets; Mt. Ma Toseong-myeon Goseong-gun, Sep. 20 2008, W. Paik NAPI-0331 (KH); Mt. Maebong Yongdae-ri Buk-myeon Inje-gun, Sep. 5-6 2008, H.-W. Kim et al. s. n. (KSU); Mt. Myeongseong Galmal-eup Cheorwon-gun, Oct. 16 2004, S.-H. Park s. n. (KH); Mt. Odae Hongcheon-gun, Aug. 9 2000, Y. Choi et al. s. n. (KH); Mt. Odae Jinbu-myeon Pyeongchang-gun, Aug. 2 1972, W. Lee s. n. (KWNU); Mt. Odae Jinbu-myeon Pyeongchang-gun, Aug. 21 1993, S. Lee et al. s. n. (SKK); Mt. Odae Jinbu-myeon Pyeongchang-gun, Jul. 28 2004, K. Yoo s. n. (KWNU); Mt. Samyeong Yanggueup Yanggu-gun, Jul. 17 2008, W. Paik NAPI-0340, NAPI-0338 (KH); Mt. Samyeong Yanggu-eup Yanggu-gun, May. 232008, W. Paik NAPI-0337 (KH); Mt. Samyeong Yanggu-eup Yanggu-gun, Sep. 5 2009, W. Paik NAPI-0341 (KH); Mt. Seorak Seorak-dong Sokcho-si, Aug. 30 2000, C. Lee et al. s. n. (SKK); Mt. Seorak Seorak-dong Sokcho-si, Jul. 27 2002, Y. Kim et al. s. n. (NIBR); Mt. Seorak Seorak-dong Sokcho-si, Jun. 27 2008, W. Paik NAPI-0256 (KH); Mt. Seorak Seorak-dong Sokcho-si, May 21 2008, W. Paik NAPI-0255 (KH); Mt. Seorak Seorak-dong Sokcho-si, Oct. 4 1983, S. Lee 19159 (SKK); Mt. Seorak Seorak-dong Sokcho-si, Oct. 9 2001, S.-H. Park et al. s. n. (KH); Mt. Seorak Seorak-dong Sokcho-si, Sep. 21 1979, G. Lee s. n. (SNU); Mt. Seorak Seorak-dong Sokcho-si, Sep. 21 1979, Y. Park s. n. (SNU); Mt. Soeppul Gwaeseok-ri Duchon-myeon Hongcheon-gun, Jul. 4 2008, W. Paik NAPI0377 (KH); Mt. Taebaek Sodo-dong Taebaek-si, Aug. 3 1976, W. Lee s. n. (KWNU); Mt. Taebaek Sodo-dong Taebaek-si, Jul. 19 2007, Kim et al. s. n. (KH) two sheets; Mt. Taebaek Sodo-dong Taebaek-si, Jun. 25 2008, Hong et al. KHUS0947 (KH); Mt. Taebaek Sodo-dong Taebaek-si, Sep. 30 2009, S. Hong et al. KHUS1799 (KH); Mt. Yonghwa Chuncheon-si, Sep. 27 2007, W. Lee et al. s. n. (KWNU); Mt. Yonghwa Hwacheon-gun, Sep. 5 2008, H.-J. LeeYonghwasan80905-1 (KH); Mt.Bangtae Inje-gun, Aug. 16 2008, W. Paik NAPI-0364 (KH); Mt.Bangtae Inje-gun, Jul. 23 2008, W. Paik s. n. (KH); Samhyeongjebong Seorak-dong Sokcho-si, Aug. 1 2008, W. Paik NAPI-0372 (KH); Samhyeongjebong Seorak-dong Sokcho-si, Aug. 1 2008, W. Paik s. n. (KH); Samhyeongjebong 
Seorak-dong Sokcho-si, Jun. 13 2008, W. Paik s. n. (KH); Sogeum River Yeongok-myeon Gangneung-si, Sep. 16 1971, T. Lee s. $n$. (SNUA); Woljeongsa Jinbu-myeon Pyeongchang-gun, Aug. 19. 1963, T. Lee et al. s. n. (SNUA); Wondae-ri Inje-eup Inje-gun, Sep. 27 2006, J.-S. Kim et al. s. n. (KSU); Gyeonggi-do: Kwangreung Namyangju-si, May 10 1959, D. Yu s. n. (SKK); Kwangreung Namyangju-si, May 27 1982, S. Lee et al. s. n. (SKK); Kwangreung Namyangju-si, May 27 1982, Y. Bae et al. s. n. (SKK); Mt. Baegun Idong-myeon Pocheon-si, Aug. 24 2002, K.-J. Kim et al. s. n. (KSU); Mt. Baegun Idong-myeon Pocheon-si, Sep. 4 2004, M.-J. Song et al. s. n. (KSU) two sheets; Mt. Chungnyeong Sudong-myeon Namyangju-si, Oct. 10 2009, Y. Ma 2010008 2010019, 2010022 2010040, 2010046, 2010050 2010065, 2010082, 2010086 (SWU); Mt. Chungnyeong Sudong-myeon Namyangju-si, Sep. 13 2002, Hong et al. s. n. (KH); Mt. Chungnyeong Sudong-myeon Namyangju-si, Sep. 19 2009, H. Lee s. n. (SWU); Mt. Eobi Seorak-myeon Gapyeong-gun, Jul. 22 2009, S.P. Hong et al. KHUS1466 (KH); Mt. Gwangdeok Dopyeong-ri Idong-myeon Pocheonsi, Sep. 10 2007, W. Paik Paik1464 (NIBR); Mt. Hwaak Hwaak-ri Buk-myeon Gapyeong-gun, Aug. 1 2006, K.-J. Kim et al. s. n. (KSU) five sheets; Mt. Hwaak Hwaak-ri Buk-myeon Gapyeong-gun, Jul. 12 2007, K. Yoo s. n. (KWNU); Mt. Hwaak Hwaak-ri Bukmyeon Gapyeong-gun, Jul. 30 2007, K. Yoo s. n. (KWNU) two sheets; Mt. Hwaak Hwaak-ri Buk-myeon Gapyeong-gun, Sep. 22 2006, H. Lee et al. s. n. (NIBR); Mt. Hwaak Hwaak-ri Buk-myeon Gapyeong-gun, Sep. 23 2006, S. Lee et al. s. n. (NIBR) two sheets; Mt. Hwaak Hwaak-ri Buk-myeon Gapyeong-gun, Sep. 9 2005, Y.-D. Kim s. n. (KSU) four sheets; Mt. Jugyeop Sohol-eup Pocheon-si, Sep. 5 2008, S.-H. Oh Jugyeopsan80905-106 (KH); Mt. Myeongji Jeongmok-ri Buk-myeon Gapyeong-gun, Aug. 1 2006, H.-T. Kim et al. s. n. (KSU); Mt. Myeongji Jeongmok-ri Buk-myeon Gapyeong-gun, Sep. 12 1981, K.-J. Kim s. n. (KSU) two sheets; Mt. Myeongji Jeongmok-ri Buk-myeon Gapyeong-gun, Sep. 9 1995, H. Roh281 (SNU); Mt. Seongnyong Buk-myeon Gapyeonggun, Sep. 10 1989, W. Lee s. $n$. (KWNU); Gyeongsangnam-do: Mt. Jiri Hadong-gun, Sep. 2 2008, Y.-K. Kim et al. s. $n$. (KSU); Mt. Jiri Hamyang-gun, Aug. 25 2004, B. Oh et al. s. n. (CBU); Mt. Jiri Hamyang-gun, Oct. 10 2004, B. Oh et al. s. n. (CBU); Mt. Jiri Hamyang-gun, Sep. 2 2008, Y.-K. Kim et al. s. n. (KSU); Mt. Jiri Hamyang-gun, Sep. 3 2008, Y.-K. Kim et al. s. n. (KSU); Mt. Jiri Sancheong-gun, Summer 1997, K.-J. Kim et al. s. n. (KSU); Gyeongsangbuk-do: Daehyeon-ri Seokpo-myeon Bonghwa-gun, Jul. 12 2008, K. Bae et al. BKH-080300 (KH); Irwol-myeon Yeongyang-gun, Sep. 20 1997, Kim\&Eah 19842 (KSU); Mt. Guryong Chunyang-myeon Bonghwa-gun, Jul. 27 2008, J. Hyun et al. 1001027 (KH); Mt. Sobaek Yeongju-si, Sep. 8 2000, K.-J. Kim et al. 1374 (KSU); Jeollanam-do: Mt. Baegyang Bukha-myeon Jangseong-gun, Oct. 25 2001, E.-S. Jeon et al. s. $n$. (KH) two sheets; Mt. Chuwol Damyang-gun, Aug. 11 1978, K.-J Kim et al. s. n. (KSU); Mt. Jiri Sandong-myeon Gurye-gun, Aug. 17 1976, W. Lee s. $n$. (KWNU); Mt. Jiri Sandong-myeon Gurye-gun, Aug. 18 2004, B. Oh et al. Guryegun(Jirisan)-040918-028 (KH) two sheets; Mt. Jiri Sandong-myeon Gurye-gun, Aug. 6 2006, K.-J. Kim et al. s. n. (KSU) three sheets; Mt. Jiri Sandong-myeon Gurye-gun, Jul. 09 2006, Y.-H. Kim et al. s. n. (KSU); Mt. Jiri Sandong-myeon Gurye-gun, Jul. 1 1913, T. Nakai s. n. (SNUA); Mt. Jiri Sandong-myeon Guryegun, Jul. 18 1961, T. Lee s. $n$. (SNUA) two sheets; Mt. Jiri Sandong-myeon Gurye-gun, Jul. 23 1963, T. Lee et al. s. n. (SNUA); Mt. Jiri Sandong-myeon Gurye-gun, Jul. 9 2006, Y.-H. Kim et al. s. n. (KSU); Mt. Jiri Sandong-myeon Gurye-gun, May 7 1994, E. Song et al. s. n. (SKK); Mt. Jiri Sandong-myeon Gurye-gun, Oct. 11 1996, S. Kim et al. s. n. (KH); Mt. Jiri Sandong-myeon Gurye-gun, Sep. 18 2004, B. Oh et al. s. n. (CBU); Mt. Jiri Sandong-myeon Gurye-gun, Sep. 24 1990, B. Lee s. n. (SNU); Mt. Jiri Sandongmyeon Gurye-gun, Sep. 24 1990, J. Kim s. n. (SNU); Mt. Jiri Sandong-myeon Gurye-gun, Sep. 24 1990, S. Kang s. n. (SNU); Mt. Jiri Sandong-myeon Gurye-gun, Sep. 24 1990, S. Lee s. n. (SNU); Mt. Jiri Sandong-myeon Gurye-gun, Sep. 25 1963, T. Lee et al. s. n. (SNUA); Mt. Jiri Sandong-myeon Gurye-gun, Sep. 9 2005, unkwon (KSU); Mt. Jiri Sandong-myeon Gurye-gun, Sep. 9 2006, J.-S. Kim et al. s. $n$. (KSU) three sheets; Mt. Mudeung Jisan-dong Dong-gu Gwangju, Aug. 1 2001, C.-H. Sin et al. s. $n$. (KH); Mt. Mudeung Jisan-dong Dong-gu Gwangju, Aug. 11 2000, H.-T. Im s. $n$. (NIBR); Jeollabuk-do: Jeongnyeongchi Jucheon-myeon Namwon-si, Aug. 7 2006, K.-J. Kim et al. s. n. (KSU); Mt. Daedun Unju-myeon Wanju-gun, Oct. 7 2004, K. Yoo et al. Wanjugun(Daedunsan)-041007-062 (KH); Mt. Deogyu Seolcheon-myeon Muju-gun, Aug. 16 2007, Kim et al. s. n. (KH); Mt. Deogyu Seolcheon-myeon Muju-gun, Aug. 17 2007, K. Heo, et al. s. n. (NIBR); Mt. Deogyu Seolcheon-myeon Muju-gun, Aug. 2 1959, T. Jung s. n. (SKK); Mt. Deogyu Seolcheon-myeon Muju-gun, Jul. 31 1987, S. Lee s. n. (SKK); Mt. Deogyu Seolcheon-myeon Mujugun, Oct. 23 2004, W. Paik s. n. (KH); Mt. Deogyu Seolcheon-myeon Muju-gun, Sep. 7 2002, J. Kim et al. $2002-0340$ (KH); Mt. Naejang Jeongeup-si, Oct. 8 2004, Y. Kim et al. s. n. (KH) two sheets; Mt. Naejang Jeongeup-si, Sep. 30 - Oct. 1 2000, B. Seon et al. 1176 (NIBR); Mt. Palgong Jangsu-eup Jangsu-gun, Aug. 28 2008, H. Lim et al. LHI1524 (KH); Mt. Palgong Jangsu-eup Jangsugun, Oct. 14 2005, C. Ho et al. CH0238 (KH); Mt. Segeol Sannae-myeon Namwon-si, Aug. 10 2005, Y.-H. Kim et al. s. n. (KSU); Mt. Segeol Sannae-myeon Namwon-si, Aug. 23 2005, K.-J. Kim et al. s. $n$. (KSU); Mt. Segeol Sannae-myeon Namwon-si, Sep. 10 2005, Y.-H. Kim et al. s. n. (KSU) three sheets; Mt. Seongsu Jangsu-gun, Jul. 3 2004, W. Paik s. n. (KH); Mt. Unjang Jinan-gun, Aug. 13 1976, W. Lee s. n. (KWNU); Chungcheongnam-do: Mt. Daedun Nonsan-si, Aug. 18 2005, W. Paik P614 (KH); 
Chungcheongbuk-do: Mt. Gakho Jodong-ri Yonghwa-myeon Yeongdong-gun, Aug. 10 2003, B. Lee et al. 1492 (NIBR); Mt. Minjuji Sangchon-myeon Yeongdong-gun, Sep. 10 2005, C.-Y. Yoon YC058 (KH); Mt. Sobaek Danyang-eup Danyang-gun, Aug. 6 1999, C.W. Park et al. s. n. (SNU); Mt. Sokri Boeun-gun, 1 1958, T. Lee s. n. (SNUA) two sheets; Mt. Sokri Boeun-gun, 1 1959, T. Lee s. n. (SNUA); Mt. Sokri Boeun-gun, Sep. 15 1966, T. Lee et al. s. n. (SNUA); Mt. Worak Jecheon-si, Sep. 22 2001, G. Park et al. s. n. (KH); Japan: Unknow, Sep. 26 1984, W. Lee s. n. (KWNU).

\section{Isodon excisus (Maxim.) Kudo f. albiflorus (Sakata) Hara 흰오리방풀}

Gyeongsangbuk-do: Mt. Guryong Chunyang-myeon Bonghwa-gun, Sep. 7 2008, M. Choi et al. s. n. (KH).

\section{Isodon inflexus (Thunb.) Kudo, L. var. inflexus 산박하}

Gangwon-do: Hoengseong-gun, Aug. 24 2004, E.-S. Jeon ESJeon41884 (KH); Mt. Chiak Wonju-si, Aug. 7 2006, K. Yoo s. n. (KWNU); Mt. Eungbong Samcheok-si, Aug. 31 2008, K. Yoo s. n. (KWNU); Samcheo-si, Jul. 22 1977, W. Lee s. n. (KWNU); Mt. Samak Chuncheon-si, Sep. 9 1973, W. Lee s. n. (KWNU); Univ. Gangwon Chuncheon-si, Sep. 15 1998, W. Lee s. n. (KWNU); Mt. Geonbong Inje-gun, Oct. 4 2000, Y.-M Lee et al. Gwang10340 (KH); Sabuk-eup Jeongseon-gun, Oct. 8 2008, H.-J. Kim et al. s. n. (KH); Guntan-ri Galmal-eup Cheorwon-gun, Jul. 23 2004, J.-M. Chung et al. 04070051 (KH); Goeun-ri Dongnae-myeon Chuncheon-si, Oct. 13 1981, S. Choi s. n. (SWU); Mt. Godae Cheorwon-gun, Oct. 10 2003, JM 03100084 (KH); Mt. Sambang Bangrim-myeon Pyeongchang-gun, Aug. 9 2001, W. Lee et al. 3120 (KSU); Mt. Baekun Maeji-ri Hungup-myon Wonju-kun, Aug. 27 1993, H. Won 93-8-53 (SNU); Mt. Bonghwa Yanggu-eup Yanggu-gun, Jul. 22 2008, W. Paik s. n. (KH); Mt. Baekun Maejiri Hungup-myon Wonju-kun, Sep. 1959, T. Lee s. n. (SNUA); Mt. Baekun Maeji-ri Hungup-myon Wonju-kun, Sep. 1960, T. Lee s. n. (SNUA); Seokgaejae Punggok-ri Gagok-myeon Samcheok-si, Sep. 18 2001, Y.-M. Lee et al. s. n. (KH); Mt. Geonbong Injegun, Oct. 4 2000, Y.-M. Lee et al. s. n. (KH); Mt. Hyangrobong Gwanin-myeon Pocheon-gun, Oct. 3 2000, Y.-M. Lee et al. s. n. (KH); Mt. soribong Gwangneung Jikdong-ri Soheul-eup Pocheon-gun, Oct. 20 2000, C.-H. Sin et al. s. n. (KH); Mt. Daeryong Chuncheon-si, Aug. 10 1975, W. Lee s. n. (KWNU); Mt. Il Hwacheon-gun, Sep. 5 2008, K. Yoo s. n. (KWNU); Sogeum River Samsan-ri Yeongok-myeon Gangneung-si, Sep. 16 1971, T. Lee s. n. (SNUA); Mt. Eungbong Samcheok-si, Oct. 2 2008, K. Yoo s. n. (KWNU); Yeongwol-eup Yeongwol-gun, Oct. 16 2002, J. Hyun et al. s. n. (KH); Mt. Maebong Chuncheon-si, Sep. 18 2008, K. Yoo s. n. (KWNU); Eungbong Jiam-ri Sabuk-myeon Chuncheon-si, Sep. 20 2007, S.-S. Kim et al. Kss0382 (KH); Eungbong Jiam-ri Sabuk-myeon Chuncheon-si, Sep. 20 2007, S.-S. Kim et al. Kss0377 (KH); Mt. Nochu Gangneung-si, Sep. 72007, J. Hyun et al. s. $n$. (KSU); Mt. Maebong Yongdae-ri Buk-myeon Inje-gun, Sep. 5-6 2008, H.-W. Kim et al. s. $n$. (KSU); Mt. Nochu Jeongseon-gun, Sep. 11 2007, J. Hyun et al. s. n. (KH); Gyeonggi-do: Mt. Chungnyeong Sudong-myeon Namyangju-si, Sep. 9 2005, Hong et al. KHU0893 (KH); Anseong-si, Sep. 2 2006, W. Lee et al. 934 (KH); Mt. Hwaya Cheongpyeong-myeon Gapyeonggun, Sep. 15 1996, S. Kim s. n. (SNU); Mt. Cheonma Onam-ri Onam-eup Namyangju-si, Oct. 6 2008, H.-T. Kim s. n. (KSU); Uijeongbu-si, Oct. 4 2003, S.-H. Park ParkSH32727 (KH); Mt. Kalbong Seungan-ri Gapyeong-eup Gapyeong-gun, Sep. 292006 , H.-T. Kim et al. s. n. (KSU); Mt. Cheonma Onam-ri Onam-eup Namyangju-si, Aug. 23 1999, Y. Lee et al. s. n. (KH); Mt. Wangbang Sineup-dong Pocheon-si, Sep. 20 1997, Y. Jeong 279 (SNU); Mt. Yongmun Yongcheon-ri Okcheon-myeon Yangpyeonggun, Sep. 28 2009, H.-W. Kim s. n. (KSU) two sheets; Mt. Cheonma Onam-ri Onam-eup Namyangju-si, Oct. 6 2008, H.-T. Kim s. $n$. (KSU); Mt. Cheonbosan Uijeongbu-si, Aug 31. 2001, E.-S. Jeon s. $n$. (KH); Mt. Taehwa Yujeong-ri Docheok-myeon Gwangjusi, Aug. 18 1983, H. Shin s. n. (SNU); Mt. Myungji Ha-myeon Gapyeong-gun, Sep. 9 1995, H. Roh 190 (SNU); Mt. Mugap Gapyeong-gun, Sep. 26 1991, C. Park at al. s. n. (SNU); Mt. Cheonma Onam-ri Onam-eup Namyangju-si, Sep. 30 1985, J. Hyun s. n. (SNU); Gwngneung Bupyeong-ri Jinjeop-eup Namyangju-si, Oct. 9 1967, Y. Lee s. n. (SKK); Mt. Chu-eup Yongmun-myeon Yangpyeong-gun, Sep. 5 2003, J. Chang et al. s. n. (SNUA); Mt. Bogae Anseong-si, Aug. 28 2002, C. Chang et al. s. n. (SNUA); Mt. Soribong Sohol-eup Pocheon-si, Sep. 18 2001, S.-H. Park s. n. (KH); Mt. Surisan Sokdal-dong Gunpo-si, Sep. 26 2005, S. Kim et al. s. n. (SKK); Mt. Hwaya Cheongpyeong-myeon Gapyeong-gun, Jun. 26 2006, W. Park s. n. (NIBR); Mt. Kalbong Seungan-ri Gapyeong-eup Gapyeong-gun, Sep. 29 2006, H.-T. Kim et al. s. n. (KSU); Gwngneung Bupyeong-ri Jinjeop-eup Namyangju-si, Sep. 26-28 1963, T. Lee et al. s. n. (SNUA) two sheets; Mt. Soyo Sangbongam-dong Dongducheon-si, Oct. 7 1990, H. Cha s. n. (SNU); Mt. Jugyeop Jikdong-ri Sohol-eup Pocheon-si, Oct. 16 2005, S.-S. Kim s. n. (KH); Namhansanseong Sanseongri Jungbu-myeon Gwangju-si, Oct. 11 2009, S.-Y. Yang s. n. (KSU); Aengjabong Usan-ri Toechon-myeon Gwangju-si, Sep. 23 2008, H.-J. Kim et al. s. n. (KH); Namhansanseong Sanseong-ri Jungbu-myeon Gwangju-si, Oct. 11 2008, S.-Y. Yang s. n. (KSU); Mt. Jugeum Sangdong-ri Sang-myeon Gapyeong-gun, Sep. 4 1984, J. Kim s. n. (SNU); Mt. Jiljang Anseong-si, Aug. 19 2006, 
W. Lee et al. 765 (KH); Sohol-eup Pocheon-si, Aug. 24 2001, E.-S. Jeon et al. Gwang10521 (KH); Pocheon-si, Sep. 111994 , G. Park s. n. (KH); Gwngneung Bupyeong-ri Jinjeop-eup Namyangju-si, Sep. 27 1975, Y. Jung s. n. (SWU); Mt. Cheonma Hwadoeup Namyangju-si, Sep. 16 2010, Y. Ma 2010077, 2010074, 2010075, 2010073, 2010066 (SWU); Mt. Sigung Yongin-si, Sep. 15 2001, Y. Kim et al. 20144 (NIBR); Mt. Gwanggyo Suwon-si, Sep. 13 2009, Y. Ma 2010020 (SWU); Mt. Wangbang Sinbuk-myeon Pocheon-si, Aug. 3 2007, W. Paik Paik1434 (NIBR); Gwngneung Bupyeong-ri Jinjeop-eup Namyangju-si, Sep. 19 1980, T. Lee et al. s. n. (SNUA); Mt. Cheonma Onam-ri Onam-eup Namyangju-si, Aug. 30 1985, J. Hyun s. n. (SNU); Mt. Myungji Ha-myeon Gapyeong-gun, Sep. 9 1995, H. Roh 190 (SNU); Mt. Sudeok Buk-myeon Gapyeong-gun, Sep. 22 2008, K.-J. Kim et al. s. n. (KH); Mt. Myeongseong Pocheon-si, Aug. 16 1989, J. Kim s. n. (SNU); Mt. Wangbang Sinbuk-myeon Pocheon-si, Sep. 7 1997, M. Yoo 248 (SNU); Mt. Surak Namyangju-si, Sep. 21 1996, J. Baek s. n. (SWU); Eumhyeon-ri Naechon-myeon Pocheon-si, Oct. 24 2006, J.-Y. Kim s. n. (KH); Mt. Jugyeop Sohol-eup Pocheon-si, Sep. 5 2008, S.-H. Oh s. n. (KH); Namhansanseong Sanseongri Jungbu-myeon Gwangju-si, Sep. 16 1981, H. Kim s. n. (SWU); Mt. Chungnyeong Sudong-myeon Namyangju-si, Oct. 10 2009, Y. Ma 2010084, 2010021 (SWU); Mt. Hwaak Buk-myeon Gapyeong-gun, Aug. 24 1985, S. Kee et al. s. n. (SKK); Gyeongsangnamdo: Ungseokbong Sancheong-eup Sancheong-gun, Aug. 8 2007, S.-H. Park et al. ParkSH73842 (KH); Mt. Beopwa Haman-gun, unknown, unknown s. n. (CBU); Hakdong-ri Dongbu-myeon Geoje-si, Jul. 23 1998, C. Lee et al. s. n. (SKK); Mt. Janggundae Munsan-eup Jinju-si, Aug. 6 1977, W. Lee s. n. (KWNU); Wahyeon-ri Irun-myeon Geoje-si, Nov. 7 2001, S.-H. Park s. n. (KH); Ungseokbong Sancheong-eup Sancheong-gun, Aug. 8 2007, S.-H. Park et al. ParkSH73836 (KH); Mt. Gaya Hapcheon-gun, Jul. 26 2003, D. Kim et al. s. n. (KH); Ugye-ri Jeokryang-myeon Hadong-gun, Oct. 15 2008, Y. Cho et al. WR-081015-125 (KH); Mt. Sinbul Eogok-dong Yangsan-si, Aug. 12 1977, W. Lee s. n. (KWNU); Mt. Jiri Macheon-myeon Hamyang-gun, Sep. 3 2008, Y.-K. Kim et al. s. n. (KSU); Gyeongsangbuk-do: Mt. Irwol Irwol-myeon Yeongyang-gun, Sep. 20 1997, Kim\&Eah 19081, 19644, 19714, 19715, 19716, 19750, 19760 (KSU); Mt. Seonam Hyeonri-ri Gaeum-myeon Uiseong-gun, Jun. 28 2008, Oh et al. 444 (KSU); Mt. Yeongdeung Ibam-myeon Yeongyang-gun, Aug. 26 1997, Kim\&Eah 19382 (KSU); Mt. Agi Imdong-myeon Andongsi, Aug. 26 1997, Kim\&Eah 19589, 19590, 19591 (KSU); Mt. Heungnim Cheonggi-myeon Yeongyang-gun, Aug. 161997, Kim\&Eah 19206 (KSU); Yean-myeon Andong-si, Aug. 26 2007, Kim\&Eah 19429, 19439, 19442 (KSU); Mt. Yeongdeung Ibammyeon Yeongyang-gun, Aug. 26 2007, Kim\&Eah 19383 (KSU); Mt. Heungnim Cheonggi-myeon Yeongyang-gun, Aug. 161997, Kim\&Eah 19207 (KSU); Yean-myeon Andong-si, Sep. 20 1997, Kim\&Eah 19780, 19781 (KSU); Mt. Gaya Suryun-myeon Seongjugun, May 22 2001, S.-H. Park et al. s. n. (KH); Gunjabong Seojung-ri Sanbuk-myeon Mungyeong-si, Sep. 18 1982, S. Hong et al. s. n. (SKK); Buk-myeon Uljin-gun, Oct. 4 2000, G. Chung at al. s. n. (MIBR); Mt. Gaya Seonju-gun, Aug. 28 2003, D. Kim et al. s. n. (KH); Mt. Yeongdeung Ibam-myeon Yeongyang-gun, Aug. 26 1997, Kim\&Eah 19405 (KSU); Mt. Maebong Mungyeongsi, Sep. 23 2000, H. Kim et al. s. n. (SNUA); Mt. Cheongnyang Gwanchang-ri Myeongho-myeon Bonghwa-gun, Aug. 15 1997, Kim\&Eah 18997 (KSU); Mt. Irwol Irwol-myeon Yeongyang-gun, Aug. 15 1997, Kim\&Eah 19037, 19047, 19048,19049 (KSU); Mt. Geumjang Uljin-gun, Aug. 14 2007, C.-Y. Chung et al. (KSU); Mt. Gaya Seongju-gun, Aug. 28 2003, D. Kim et al. s. n. (KH); Mt. Hwanghak Giran-myeon Andong-si, Aug. 29 1993, J. Park s. n. (SNU); Mt. Guryong Chunyang-myeon Bonghwagun, Aug. 16 2008, S. Kang et al. 1101020 (KH); Mt. Guryong Chunyang-myeon Bonghwa-gun, Sep. 7 2008, Y. Oh et al. 1202022 (KH); Mt. Eungbong Buk-myeon Uljin-gun, Oct. 4 2000, G. Chung et al. s. n. (NIBR); Mt. Cheongnyang Gwanchang-ri Myeonghomyeon Bonghwa-gun, Aug. 15 1997, Kim\&Eah 18996 (KSU); Busan: Mt. Hwangnyeong Yeonje-gu, Oct. 1 1990, J. Kim s. n. (SNU); Seoul: Cheongnyangni Jeonnong-dong Dongdaemun-gu, Aug. 30 2001, C.-W. Park et al. s. n. (SNU); Mt. Bukhan Gugidong Jongno-gu, Sep. 8 2000, J. Moon s. n. (SNU); Mt. Bukhan Jeongneung-dong Seongbuk-gu, Oct. 15 1961, W. Lee s. n. (SKK); Mt. Gwanak Gwanak-gu, Oct. 1984, I. Kim s. n. (KH); Myeongnyundong Jongno-gu, Sep. 13 1958, K. Moon s. n. (SKK); Incheon: Bukpo-ri Baengnyeong-myeon Ongjin-gun, Oct. 6 1965, T. Lee et al. s. n. (SNUA); Jeondeungsa Onsu-ri Gilsang-myeon Ganghwa-gun, Sep. 11 1976, H. Park s. n. (SWU); Mt. Goryeo Naega-myeon Ganghwa-gun, Aug. 31 2005, S. Kwon 3491 (NIBR); Mt. Haemyeong Seongmo-ri Samsan-myeon Ganghwa-gun, Sep. 8 2007, C.-S. Chang et al. s. n. (NIBR); Mt. Hwagae Eumnaeri Gyodong-myeon Ganghwa-gun, Sep. 1 2005, S. Kwon et al. s. n. (NIBR); Mt. Mani Hwado-myeon Ganghwa-gun, Aug. 30 2003, K.-J. Kim et al. s. n. (KSU) two sheets; Songhae-myeon Ganghwa-gun, Aug. 17 2004, W. Lee et al. 1536 (KH); Jeollanamdo: Mt. hyeongjebong Godal-myeon Gokseong-gun, Aug. 26 2005, T.-G. No et al. 283 (SNU); Bongdeok-ri Dodeok-myeon Goheung-gun, Oct. 1 2008, Y. Cho at al. WR-081001-152 (KH); Daesin-ri Baeksu-eup Yeonggwang-gun, Oct. 1 2006, H.-C. Shin et al. s. n. (NIBR); Deokheung-ri Hyeonsan-myeon Haenam-gun, Jul. 12 2006, Y. Cho at al. WR-060712-167 (KH); Gagok-dong Suncheon-si, Sep. 16 2009, Y. Cho WR-090916-182 (KH); Geomundo Samsan-myeon Yeosu-si, Oct. 20 2002, Y.-M. Kim et al. L-60399 (KH); Geomundo Samsan-myeon Yeosu-si, Oct. 20 2002, Y.-M. Kim et al. s. n. (KH); Jungdo-ri Wando-eup Wando-gun, 
Oct. 3 2003, K. Heo et al. s. n. (SKK); Mt. Baegun Gurye-gun, unknown, unknown s. n. (CBU); Mt. Cheonbong Boknae-myeon Boseong-gun, Aug. 11 2005, H.-J. Choi et al. CHJ\&NGH50397 (KH); Mt. Chuwol Damyang-gun, Aug. 11 1978, K.-J. Kim et al. s. n. (KSU); Mt. Dojisan Bukha-myeon Jangseong-gun, Aug. 17 2004, H. Im Jangseong-04817-483 (KH); Mt. Duryun Samsanmyeon Haenam-gun, Sep. 15 1993, H. Yi s. n. (SNU); Mt. Duryun Samsan-myeon Haenam-gun, Aug. 1993, H. Yi s. n. (SNU); Mt. Gubonghwa Gwangyang-eup Gwangyang-si, Oct. 8 2004, B. Oh et al. s. n. (KH); Mt. Jiri Gurye-gun, unknown, unknown s. n. (CBU); Mt. Jiri Naedong-ri Toji-myeon Gurye-gun, Sep. 25 1990, H. Noh s. n. (SWU); Mt. Malbong Hwasun-gun, Aug. 10 2005, S.-H. Park ParkSH52636 (KH); Mt. Mohu Yuma-ri Nam-myeon Hwasun-gun, Sep. 7 2006, Hong et al. s. n. (NIBR); Mundeok-myeon Boseong-gun, Oct. 12. 2005, S.H. Park ParkSH53720 (KH); Seongnamdo-ri Jodo-myeon Jindo-gun, Jul. 28 2002, J. Hyun et al. 2002236 (KH); Jeollabuk-do: Mt. Byeonsan Buan-gun, Jul. 31 1963, Chung\&Lee s. n. (SKK); Mt. Cheondeung Sanbuk-ri Unju-myeon Wanju-gun, Oct. 3 2008, I. Lim LHI1727 (KH); Mt. Deogyu Seolcheon-myeon Muju-gun, Apr. 4 2002, J. Kim et al. s. n. (KH); Mt. Deogyu Seolcheon-myeon Muju-gun, 29 Jul. 2008, H. Kim 4634 (KH); Mt. Minjuji Muju-gun, Aug. 11 1976, W. Lee s. n. (KWNU); Jeju-do: Gosan-ri Hangyeong-myeon Jeju-si, Sep. 19 2006, J.-S. Kim et al. s. n. (KSU); Jungmundong Seogwipo-si, Oct. 9 1993, S.-H. Park et al. s. n. (KSU); unknwon, Jul. 22 1954, T. Chung s. n. (SKK); Chungcheongnamdo: Cheollipo Seosan-si, Sep. 26 1980, M. Lee s. n. (SKK); Mt. Baekam Geonchun-ri Nami-myeon Geumsan-gun, Aug. 24 2008, H. Lee s. n. (KH) two sheets; Mt. Chilgap Cheongyang-gun, Aug. 7 2000, Oh et al. 392 (NIBR); Mt. Chilgap Cheongyang-gun, Sep. 3 2000, Oh et al. 444 (NIBR); Mt. Dogo Sijeon-ri Dogo-myeon Asan-si, Oct. 12 2008, E.-S. Jeon s. n. (KH); Mt. Gyeryong Nonsan-si, Sep. 2 2008, H. Lee HNU2254 (KH); Mt. Jinak Geumsan-eup Geumsan-gun, Oct. 15 2006, B. Oh et al. s. n. (KH); Mt. Oseo Cheongna-myeon Boryeong-si, Oct. 2 1997, S. Yong 75 (SNU); Mt. Oseo Cheongna-myeon Boryeong-si, Oct. 2 1997, Y. Cho 76 (SNU); Mt. Oseo Cheongna-myeon Boryeong-si, Sep. 11 2007, H. Lee HNU1718 (KH); Mt. Oseo Cheongna-myeon Boryeong-si, Oct. 2 1997, K. Seo 98 (SNU); Mt. Taejo Anseo-dong Cheonan-si, Sep. 19 2007, H. Lee s. n. (KH); Mt. Taejo Dongnam-gu Cheonan-si, Sep. 16 1988, M. Kim s. n. (SNU); Sinjindo-ri Geunheung-myeon Taean-gun, Oct. 1 2007, S.-H. Park et al. s. n. (KH); unknwon, Oct. 5 1955, S.-H. Park s. n. (SKK); unknwon, Jul. 15 1963, S.-H. Park s. n. (SKK); Chungcheongbukdo: Mt. Cheongwan Hangok-ri Yongsan-myeon Yeongdong-gun, Jun. 29 2005, J. Kim et al. s. n. (NIBR); Mt. Cheontae Yeongdonggun, Aug. 13 2006, H.-J. Choi et al. CHJ60263 (KH); Mt. Hwangak Sangchon-myeon Yeongdong-gun, Jul. 17 2005, C.-Y. Yoon YC095 (KH); Mt. Jakseong Geumseong-myeon Jecheon-si, Aug. 14 2006, G.-H Nam et al. s. n. (KH) three sheets; Mt. Ogap Eumseong-gun, Sep. 24 2005, I. Kwon san80 (KH); Mt. Worak Hansu-myeon Jecheon-si, Aug. 23 2010, Y. Ma 2010085 (SWU); Mt. Worak Hansu-myeon Jecheon-si, Oct. 10 2002, Y.-M. Kim et al. (KH); Nogobong Deunggok-ri Buyong-myeon Cheongwongun, Sep. 9 2005, K. Yoo s. n. (KWNU); Seongnae-ri Geumseong-myeon Jecheon-si, Oct. 18 2006, S.-H. Park ParkSH63627 (KH); unknwon, Aug. 19 1988, J. Lee s. n. (SNU); UnKnown: unknwon, Jun. 28 1982, H. Kim s. n. (SKK).

\section{Isodon inflexus (Thunb.) Kudo, L. var. canescens (Nakai) Kudo 털산박하}

Gangwon-do: Mt. Chorokbong Donghae-si, Aug. 31 2008, K. Yoo s. n. (KWNU); Gangchonri Namsan-myeon Chuncheon Si, Sep. 6 1981, S. Choi s. n. (SWU); Mt. Daeryong Dongnae-myeon Chuncheon-si, Aug. 10 1975, W. Lee s. n. (KWNU); GyeonggiDo: Namhansanseong Sanseong-ri Jungbu-myeon Gwangju Si, Oct. 11 2008, S.-Y. Yang s. n. (KSU); Mt. Cheonggye Gwacheon Si, Sep. 12 1987, S. Gu s. n. (SWU); Gyeongsangnam-do: Mt. Jeongjok Ungchon-myeon Ulju-gun Ulsan, Sep. 23 2000, K.-J. Kim et al. s. n. (KSU); Mt. Mangun Seo-myeon Namhae-gun, Aug. 11 1955, T. Chung s. n. (SKK); Jeollanam-do: Hongdo-ri Heuksan-myeon Sinan-gun, Sep. 18 2006, E.-S. Jeon ESJeon61592 (KH); Jindo-gun, Aug. 1 1956, T. Chung s. n. (SKK); Mokposi, Jul. 28 1986, T. Chung s. n. (SKK); Seongnamdo Jindo-gun, Jul. 28 2002, J. Hyun et al. s. n. (KH); Mt. Dalma Haenam-gun, Oct. 27 2005, S.-H. Park ParkSH54174 (KH); Jeollabuk-do: Mt. Soyo Buan-myeon Gochang-gun, Aug. 9 2006, B.-Y. Sun 0549 (NIBR); Jeju-do: Chujado Chuja-myeon Jeju-si, unknwon, T. Lee $s . n$. (SNUA) nine sheets; Mt. Halla Jeju-si, Oct. 3 1966, T. Lee et al. s. $n$. (SNUA) three sheets; Unknow, Jul. 22 1954, T. Chung s. n. (SKK); Chungcheongnam-do: Mt. Taejo Dongnamgu Cheonan-Si , Sep. 16 1988, M. Kim s. n. (SNU); Chungcheongbuk-do: Cheongju-si, Jul. 5 1953, D. No s. n. (SKK).

\section{Isodon inflexus (Thunb.) Kudo, L. var. microphyllus (Nakai) Kudo 영도산박하}

Gyeongsangnam-do: Samdong-myeon Namhae-gun, Oct. 18 2007, H.-J. et al. ChoiHJ70494 (SNUA); Ugye River Ugye-ri Jeongnyang-myeon Hadong-gun, Oct. 15 2008, Y. Cho et al. WR-081015-126 (KH); Jeollanam-do: Bogildo Bogil-myeon Wando-gun, Aug. 13 1964, T. Lee et al. s. n. (SNUA) three sheets; Jeongjamaeul Jeongja-ri Bogil-myeon Wando-gun, Oct. 18 2006, Y. Cho et al. WR-061018-021 (KH); Jeju-do: Bijarim Gujwa-eup Jeju-si, Oct. 4 1966, T. Lee et al. s. n. (SNUA); Unknow, unknwon, T. Lee s. $n$. (KH). 


\section{Isodon japonicus (Burmann) Hara 방아풀}

Gangwon-do: Buksan-myeon, Sep. 2 2005, K. Yoo s. n. (KWNU); Buksan-myeon Chuncheon-si, Aug. 18 2006, K. Yoo s. n. (KWNU); Chugok-ri Buksan-myeon Chuncheon-si, Jun. 16 2004, J.-M. Chung et al. (KH); Daei-ri Singi-myeon Samcheok-si, Aug. 26 1995, J.-i. Jeon s. n. (SNUA); Inje-gun, Sep. 4 2000, K. Yang et al. s. n. (NIBR); Mitan-myeon Pyeongchang-gun, Sep. 13 2006, E.-S. Jeon ESJeon61653 (KH); Mt. Baekun Maeji-ri Heungeop-myeon Wonju-si, Aug. 27 1993, H. Won 93-8-42 (SNU); Mt. Bangtae Girin-myeon Inje-gun, Sep. 18 2008, W. Paik s. n. (KH); Mt. Deokhangsan Samcheok-si, Sep. 24 2005, G. Kim et al. s. n. (KH); Mt. Deokhangsan Samcheok-si, Sep. 4 2005, J. Hyun et al. s. n. (KH); Mt. Duta Hajang-myeon Samcheok-si, Sep. 8 2007, J. Hyun et al. s. n. (KH) two sheets; Mt. Duta Miro-myeon Samcheok-si, Oct. 7 2007, J. Hyun et al. s. n. (KH); Mt. Eungbong Samcheok-si, Aug. 30 2008, K. Yoo s. n. (KWNU); Mt. Gari Duchon-myeon Hongcheon-gun, Oct. 2 1977, W. Lee s. n. (KWNU); Mt. Geombong Samcheok-si, Oct. 15 2008, K. Yoo s. n. (KWNU); Mt. Gongjak Dong-myeon Hongcheon-gun, Sep. 4 1979, W. Lee s. n. (SKK); Mt. Jieok Nam-myeon Jeongseon-gun, Aug. 11 1999, W. Lee s. n. (KWNU); Mt. Mandeokbong Gangneung-si, Sep. 1 1996, W. Lee s. $n$. (KWNU) four sheets; Mt. Myeun, Sep. 16 1990, S.-H. Oh s. n. (SNU); Mt. Samak Seomyeon Cheorwon-gun, Oct. 8 1988, W. Lee s. n. (KWNU); Mt. Seokbyeong Okgye-myeon Gangneung-si, Aug. 13 2006, J. Hyun et al. s. n. (KH) two sheets; Mt. Seokbyeong Okgye-myeon Gangneung-si, Aug. 22 2002, C.-W. Park et al. s. n. (SNU); Mt. Seokbyeong Okgye-myeon Gangneung-si, Aug. 27 2006, J. Hyun et al. s. n. (KH) two sheets; Mt. Seokbyeong Okgye-myeon Gangneung-si, Sep. 9 2006, J. Hyun et al. s. n. (KH); Mt. Yonghwa Sabuk-myeon Chuncheon-si, Sep. 19 1976, Y. Kil s. n. (KWNU); Munhye-ri Galmal-eup Cheorwon-gun, Aug. 20 2008, E.-S. Jeon et al. ESJeon81728, 81729 (KH); Surimji Wonju-si, Sep. 19 1976, W. Lee s. n. (KWNU); Gyeonggi-do: Mt. Cheonma Hwado-eup Namyangju-si, Oct. 6 2008, H.-T. Kim s. n. (KSU); Mt. Cheonma Hwado-eup Namyangju-si, Sep. 16 2010, Y. Ma 2010007, 2010047, 2010066, 2010071, 2010072, 2010076 (SWU); Mt. Cheonma Hwado-eup Namyangju-si, Sep. 30 1985, J. Hyun s. n. (SNU); Mt. Chungnyeong Sudong-myeon Namyangju-si, Oct. 10 2009, Y. Ma 2010048, 2010049 (SWU); Mt. Gwanggyo Suwon-si, Sep. 13 2009, Y. Ma 2010020 (SWU); Mt. Jeonggwang Docheok-myeon Gwangju-si, Oct. 10 2009, Y. Ma 2010069, 2010086 (SWU); Mt. Maguk Seolseong-myeon Icheon-si, Sep. 18 2002, J. Hyun et al. s. n. (NIBR); Mt. Namhansanseong Gwangju-si, Sep. 30 2010, Y. Ma 2010067, 2010068, 2010070, 2010078, 2010079, 2010080, 2010081, 2010082, 2010083, 2010084, 2010085, 2010086 (SWU); Mt. Suri Sokdal-dong Gunpo-si, Sep. 26 2005, S. Kim et al. s. n. (SKK); Mt. Taehwa Gwangju-si, Jul. 7 2000, D. Choi et al. s. n. (KH); Toechon-myeon Gwangju-si, Sep. 24 2008, S.-H. Park ParkSH82224 (KH); Gyeongsangnam-do: Mt. Janggundae Munsan-eup Jinju-si, Aug. 6 1977, S. Lee s. $n$. (KWNU); Gyeongsangbuk-do: Mt. Geumjangsan Oeseonmi-ri Onjeong-myeon Uljin-gun, Oct. 7 2007, G.-Y. Chung s. n. (NIBR); Mt. Hakga Yechon-gun, Sep. 21 2003, G.-Y. Chung at al. s. n. (KH); Mt. Hakgasan Sanseong-ri Bomun-myeon Yecheongun, Sep. 25 2006, B. Oh et al. s. n. (KH); Mt. Irwol Irwol-myeon Yeongyang-gun, Aug. 18 2006, G.-Y. Jeong s. n. (KH); Mt. Irwol Irwol-myeon Yeongyang-gun, Oct. 22 1961, W. Lee s. n. (SKK); Mt. Noeum Namjang-dong Sangju-si, Sep. 22005 , C. Chang Sky0919 (NIBR); Mt. Seonyusan Oeseonmi-ri Onjeong-myeon Uljin-gun, Oct. 7 2007, G.-Y. Chung et al. s. n. (NIBR); Mt. Sobaek Punggi-eup Yeongju-si, Aug. 16 1989, W. Lee s. n. (KWNU); Jeollabuk-do: Mt. Deogyu Seolcheon-myeon Mujugun, Sep. 28 2003, D. Kim s. n. (KH); Chungcheongnam-do: Mt. Dogo Dogo-myeon Asan-si, Oct. 12 2008, E.-S. Jeon s. n. (KH); Chungcheongbuk-do: Maepo-eup Danyang-gun, Aug. 27 1965, T. Lee et al. s. n. (SNUA) two sheets; Mt. Cheondeung Sancheok-myeon Chungju-si, May 14 2002, S. Ko et al. s. n. (NIBR); Mt. Duak Danyang-gun, Oct. 9 1959, W. Lee s. n. (SKK); Mt. Duta Jincheon-gun, Aug. 26 2003, W. Paik 3861 (NIBR); Mt. Garibong Noeun-myeon Chungju-si, Aug. 3 1989, H. Lee s. n. (SNU); Mt. Oksunbong Danyang-gun, Oct. 10 1959, W. Lee s. n. (SKK); Mt. Worak Danseong-myeon Danyang-gun, Sep. 24 2005, C.-G. Jang 298 (NIBR); Mt. Worak Hansu-myeon Jecheon-si, Aug. 23 2010, Y. Ma 2010001, 2010002, 2010003, 2010004, 2010005, 2010006, 2010041, 2010042, 2010043, 2010044, 2010045 (SWU); Mt. Worak Hansu-myeon Jecheon-si, Sep. 24 2001, K. Park et al. s. n. (KH); China: Shaanxi Baoji city forest near from Panjiawan, Aug. 31 2008, C.-S. Chang SX1856, 1857 (KH).

\section{Isodon serra (Maximowicz) Kudo 자주방아풀}

Gangwon-do: Mt. Gariwang Jeongseon-eup Jeongseon-gun, Sep. 7 1989, H. Lee s. n. (SNU); Mt. Geumbyeong Sindong-myeon Chuncheon-si, Aug. 10 1979, W. Lee s. n. (KWNU); Mt. Seokbyeong San-gye-ri Okgye-myeon Gangneung-si, Apr. 13 2002, C.-W. Park et al. s. n. (SNU) three sheets; Mt. Seokbyeong San-gye-ri Okgye-myeon Gangneung-si, Aug. 22 2002, C.-W. Park et al. s. $n$. (SNU); Mt. Seongjubong Yanggu-gun, Sep. 6 2008, K. Yoo s. $n$. (KWNU); Mt. Taehwa Yeongwol-gun, Sep. 24 2001, Oh et al. 581 (NIBR); MT. Yukbaek Dogye-eup Samcheok-si, Aug. 31 2007, G. Chung ANH-en-070831-011 (NIBR); Gyeongsangnam-do: Hapcheon-gun, Sep. 18 2002, H. Shin et al. s. n. (NIBR); Jeollanam-do: Gwangju, Aug. 26 2001, E.-S. Jeon et al. s. n. (KH) four sheets. 